\title{
THE SDSS-III APOGEE RADIAL VELOCITY SURVEY OF M DWARFS. I. DESCRIPTION OF THE SURVEY AND SCIENCE GOALS
}

\author{
R. Deshpande ${ }^{1,2}$, C. H. Blake ${ }^{3,4}$, C. F. Bender ${ }^{1,2}$, S. Mahadevan ${ }^{1,2}$, R. C. Terrien ${ }^{1,2}$, J. K. CarlberG ${ }^{5}$, G. Zasowski ${ }^{6,7}$, \\ J. Crepp ${ }^{8}$, A. S. Rajpurohit ${ }^{9}$, C. Reylé ${ }^{9}$, D. L. Nidever ${ }^{10}$, D. P. Schneider ${ }^{1,2}$, C. Allende Prieto ${ }^{11,12}$, D. Bizyaev ${ }^{13}$, \\ G. Ebelke ${ }^{14}$, S. W. Fleming ${ }^{1,2}$, P. M. Frinchaboy ${ }^{15}$, J. GE ${ }^{16}$, F. Hearty ${ }^{6}$, J. Hernández ${ }^{11,12}$, E. Malanushenko ${ }^{13}$, \\ V. Malanushenko ${ }^{13}$, S. R. Majewski ${ }^{6}$, R. Marchwinski ${ }^{2}$, D. Muna ${ }^{16}$, D. Oravetz ${ }^{13}$, K. Pan $^{13}$, R. P. Schiavon $^{17}$, \\ M. Shetrone ${ }^{18}$, A. Simmons ${ }^{13}$, K. G. Stassun ${ }^{19,20}$, J. C. Wilson ${ }^{6}$, And J. P. Wisniewski ${ }^{21}$ \\ ${ }^{1}$ Center for Exoplanets and Habitable Worlds, The Pennsylvania State University, University Park, PA 16802, USA \\ ${ }^{2}$ Department of Astronomy and Astrophysics, The Pennsylvania State University, University Park, PA 16802, USA \\ ${ }^{3}$ Department of Physics and Astronomy, University of Pennsylvania, Philadelphia, PA 19104, USA \\ ${ }^{4}$ Department of Astrophysical Sciences, Princeton University, Peyton Hall, Ivy Lane, Princeton, NJ 08544, USA \\ ${ }^{5}$ Department of Terrestrial Magnetism, Carnegie Institution of Washington, 5241 Broad Branch Road NW, Washington, DC 20015, USA \\ ${ }^{6}$ University of Virginia, 530 McCormick Road, Charlottesville, VA 22904, USA \\ ${ }^{7}$ Department of Physics and Astronomy, Johns Hopkins University, 3400 North Charles Street, Baltimore, MD 21218, USA \\ ${ }^{8}$ Department of Physics, University of Notre Dame, 225 Nieuwland Science Hall, Notre Dame, IN 46556, USA \\ ${ }^{9}$ Institut UTINAM, CNRS UMR 6213, Observatoire des Sciences de l'Univers THETA Franche-Comt é-Bourgogne, \\ Université de Franche Comté, Observatoire de Besançon, BP 1615, F-25010 Besançon Cedex, France \\ ${ }^{10}$ Department of Astronomy, University of Michigan, Ann Arbor, MI 48109, USA \\ ${ }^{11}$ Instituto de Astrofísica de Canarias, E-38205 La Laguna, Tenerife, Spain \\ ${ }^{12}$ Departamento de Astrofísica, Universidad de La Laguna, E-38206 La Laguna, Tenerife, Spain \\ ${ }^{13}$ Apache Point Observatory, P.O. Box 59, Sunspot, NM 88349-0059, USA \\ ${ }^{14}$ Department of Physics and Astronomy, Texas Christian University, TCU Box 298840, Fort Worth, TX 76129, USA \\ ${ }^{15}$ Department of Astronomy, University of Florida, 211 Bryant Space Science Center, Gainesville, FL 32611-2055, USA \\ ${ }^{16}$ Department of Astronomy, Ohio State University, Columbus, OH 43210, USA \\ ${ }^{17}$ Astrophysics Research Institute, Liverpool John Moores University, Wirral, CH41 1LD, UK \\ ${ }_{18}$ McDonald Observatory, The University of Texas at Austin, Austin, TX 78712, USA \\ ${ }^{19}$ Department of Physics and Astronomy, Vanderbilt University, Nashville, TN 37235, USA \\ ${ }^{20}$ Department of Physics, Fisk University, Nashville, TN, USA \\ ${ }^{21}$ Astronomy Department, University of Washington, Box 351580, Seattle, WA 98195, USA \\ Received 2013 July 26; accepted 2013 October 1; published 2013 November 8
}

\begin{abstract}
We are carrying out a large ancillary program with the Sloan Digital Sky Survey, SDSS-III, using the fiber-fed multiobject near-infrared APOGEE spectrograph, to obtain high-resolution $H$-band spectra of more than $1200 \mathrm{M}$ dwarfs. These observations will be used to measure spectroscopic rotational velocities, radial velocities, physical stellar parameters, and variability of the target stars. Here, we describe the target selection for this survey, as well as results from the first year of scientific observations based on spectra that will be publicly available in the SDSS-III DR10 data release. As part of this paper we present radial velocities and rotational velocities of over $200 \mathrm{M}$ dwarfs, with a $v \sin i$ precision of $\sim 2 \mathrm{~km} \mathrm{~s}^{-1}$ and a measurement floor at $v \sin i=4 \mathrm{~km} \mathrm{~s}^{-1}$. This survey significantly increases the number of $M$ dwarfs studied for rotational velocities and radial velocity variability (at $\sim 100-200 \mathrm{~m} \mathrm{~s}^{-1}$ ), and will inform and advance the target selection for planned radial velocity and photometric searches for low-mass exoplanets around M dwarfs, such as the Habitable Zone Planet Finder, CARMENES, and TESS. Multiple epochs of radial velocity observations enable us to identify short period binaries, and adaptive optics imaging of a subset of stars enables the detection of possible stellar companions at larger separations. The high-resolution APOGEE spectra, covering the entire $H$ band, provide the opportunity to measure physical stellar parameters such as effective temperatures and metallicities for many of these stars. At the culmination of this survey, we will have obtained multi-epoch spectra and radial velocities for over 1400 stars spanning the spectral range M0-L0, providing the largest set of near-infrared $\mathrm{M}$ dwarf spectra at high resolution, and more than doubling the number of known spectroscopic $v \sin i$ values for M dwarfs. Furthermore, by modeling telluric lines to correct for small instrumental radial velocity shifts, we hope to achieve a relative velocity precision floor of $50 \mathrm{~m} \mathrm{~s}^{-1}$ for bright $\mathrm{M}$ dwarfs. With three or more epochs, this precision is adequate to detect substellar companions, including giant planets with short orbital periods, and flag them for higher-cadence followup. We present preliminary, and promising, results of this telluric modeling technique in this paper.
\end{abstract}

Key words: binaries: spectroscopic - instrumentation: spectrographs - methods: observational - stars: fundamental parameters - stars: low-mass - techniques: radial velocities

Online-only material: color figures, machine-readable and VO tables

\section{INTRODUCTION}

M dwarfs are a major stellar constituent of the Galaxy and are increasingly important for exoplanet science and the quest for discovering low-mass planets in or near habitable zones (Dressing \& Charbonneau 2013; Kopparapu 2013). Highresolution spectroscopic observations of $\mathrm{M}$ stars are crucial for understanding stellar astrophysics at the bottom of the main sequence and planet statistics across the H-R diagram. A large, multi-epoch spectroscopic survey enables us to not only detect 
low-mass stellar and sub-stellar companions (including giant planets) but also to address a wide range of questions, including the statistics of stellar multiplicity, kinematics, metallicity, and activity. High-resolution spectra in the near infrared (NIR) for a range of $\mathrm{M}$ dwarfs will also be critical for modeling of chemical abundances and for probing the physical processes that occur in the complex atmospheres of $\mathbf{M}$ dwarfs. The multiplexed Sloan Digital Sky Survey (SDSS-III) Apache Point Observatory Galactic Evolution Experiment (APOGEE) spectrograph is uniquely suited to provide many hundreds of such spectra, increasing the number of available high-resolution NIR M dwarf spectra by at least an order of magnitude.

Radial velocity (RV) surveys to detect exoplanets have mostly targeted FGK stars over the past two decades, but are beginning to explore the $\mathrm{M}$ dwarfs in the solar neighborhood. With temperatures below $\sim 4000 \mathrm{~K}$, the spectral energy distributions of M dwarfs peak between 0.9 and $1.8 \mu \mathrm{m}$ (the $Y, J$, and $H$ bands), where RV measurement techniques are less developed than in the optical. Rodler et al. (2012) derived RV measurements of eight late-M dwarfs as part of a large survey by Deshpande et al. (2012) to monitor RV variability among late-M dwarfs (M5.0-M9.5) using the NIRSPEC spectrograph (McLean et al. 1998). At a spectral resolution $R \sim 20,000$, in the $J$ band, they obtained RV precisions between $180 \mathrm{~m} \mathrm{~s}^{-1}$ and $300 \mathrm{~m} \mathrm{~s}^{-1}$. Blake et al. (2010) targeted a magnitude-limited sample of M dwarfs and ultracool dwarfs using NIRSPEC over a period of $6 \mathrm{yr}$. Employing a forward-modeling technique in the $K$ band (Blake et al. 2007) at $R \sim 25,000$, they report an RV precision of $50 \mathrm{~m} \mathrm{~s}^{-1}$ for a bright, slowly rotating $\mathrm{M}$ dwarf and $200 \mathrm{~m} \mathrm{~s}^{-1}$ for slowly rotating, ultracool dwarfs. Bean et al. (2010) used ammonia gas cell as an iodine analog in the NIR on the very high ( $R \sim 100,000)$ spectrograph CRIRES (Kaüfl et al. 2004) in the $K$ band and achieved an RV precision of $\sim 3-5 \mathrm{~m} \mathrm{~s}^{-1}$ over long timescales.

Though this level of precision is a big step forward, it is restricted to wavelength regions overlapping the ammonia cell and for relatively bright stars. In addition, the process also requires very high spectral resolution, which is not generally available. New infrared instruments like Habitable Zone Planet Finder (Mahadevan et al. 2012) and CARMENES (Quirrenbach et al. 2010) are now being designed to try to detect Earth-mass planets in the habitable zones of nearby low-mass $M$ dwarfs. Target selection for these surveys (which tend to be very expensive in terms of telescope time) will benefit immensely from the characterization of activity and binarity in a large sample of M dwarfs.

Fully convective, late-type $\mathrm{M}$ dwarfs show strong magnetic activity (Morin et al. 2008; Browning 2008; Reiners \& Basri 2008, 2009, 2010). A relation between stellar activity and projected rotational velocity $(v \sin i)$ is well established for FGK stars (Noyes 1984) and appears to govern the M dwarfs as well (Reiners et al. 2012). Therefore, $v \sin i$ acts as a good proxy for activity. In general, fast rotating $M$ dwarfs are more active than their slow rotating counterparts. Reiners \& Basri (2008) showed that early-M dwarfs are slow rotators while stars M5 and later, a boundary where they become fully convective (Chabrier \& Baraffe 1997), are generally fast rotators ( $\left.v \sin i>10 \mathrm{~km} \mathrm{~s}^{-1}\right)$. More recently Irwin et al. (2011) published rotation periods for 41 field $\mathrm{M}$ dwarfs from the MEarth survey (Nutzman \& Charbonneau 2008) showing a fast rotating group and a slowly rotating group. This photometric survey is able to detect the slowly rotating group that falls below the sensitivity threshold of RV surveys.
A study of a sample of $27 \mathrm{M}$ dwarfs over a period of $6 \mathrm{yr}$ by Gomes da Silva et al. (2012) found RV variations with a amplitudes $\geqslant 5 \mathrm{~m} \mathrm{~s}^{-1}$ among $36 \%$ of their stars, which they attributed to magnetic cycles. Therefore, measuring $v \sin i$ to identify slowly rotating stars that are likely less active (or at least likely to exhibit less RV noise) is an important component of any search for low-mass companions to low-mass stars.

RV surveys of solar-type stars have estimated the frequency of giant planets at $\sim 7 \%$ for FGK stars (Marcy et al. 2000; Udry et al. 2007) within 10 AU. The frequency of brown dwarf (BD) companions in RV surveys, however, falls down to about $0.6 \%-1 \%$ at similar orbital distances (Marcy \& Butler 2000; Grether \& Lineweaver 2006). When restricting the sample to "hot Jupiters" (at $a<0.1 \mathrm{AU}$ ), the frequency of giant planets is $\sim 1.2 \%$, and $2.5 \%$ for $a<1 \mathrm{AU}$. The expected frequency of close-in giant planets ( $a<1 \mathrm{AU})$ from the M-dwarf survey at the Hobby-Eberly Telescope (Endl et al. 2003) is roughly $<1.3 \%$ (Endl et al. 2006).

On the other hand, Metchev \& Hillenbrand (2009) found a frequency of $3.2 \%$ of BDs from an adaptive optics (AO) survey of 266 young solar type stars at larger distances between 28-1590 AU, and Lafrenière et al. (2007) found a frequency of $1.9 \%$ of BDs companion at $\sim 25-250$ AU. Jódar et al. (2013) have found a volume-limited binary fraction of early-to-midtype $\mathrm{M}$ dwarfs of $8.8 \%$ from a sample of $451 \mathrm{M}$ dwarfs at less than $25 \mathrm{pc}$ (at orbital distances less than $80 \mathrm{AU}$ ). When including literature data at larger orbital distances these authors find a binary fraction of $\sim 20.3 \%$.

Exploring these large orbital distances is probably beyond the capabilities of the APOGEE M-Dwarf Survey, but we will be able to discover close-in BDs around M dwarfs, and therefore place constraints on the frequency of close-in BDs and maybe giant planets around $\mathrm{M}$ dwarfs.

The well-established correlation between metallicity and giant planet occurrence seems to also apply to M dwarfs (Neves et al. 2013; Rojas-Ayala et al. 2012; Terrien et al. 2012b). The difference between mean metallicity of the M-dwarf stars with and without giant planets is 0.20 dex (Neves et al. 2013), from a sample of $102 \mathrm{M}$ dwarfs in the HARPS RV survey (Bonfils et al. 2013).

Imaging and RV observations of stellar multiple systems enable direct measurements of the physical properties of stars and provide a key window into the process of star formation. Comprehensive surveys probing a wide range of orbital separations using multiple observational techniques have been carried out for F, G, and K stars (Duquennoy \& Mayor 1991; Raghavan et al. 2010), but sample sizes for similar surveys targeting low-mass stars remain small (Fischer \& Marcy 1992; Marchal et al. 2003; Delfosse et al. 2004). There are, however, clear indications that the overall rate of occurrence of multiple systems is a strong function of stellar mass (Lada 2006; Raghavan et al. 2010). Using a combination of Doppler velocity and direct imaging techniques Montet et al. (2013) find that $\sim 6.5 \%$ of all $\mathrm{M}$ dwarfs stars host a giant planet with $1<M_{J}<13 M_{J}$ and $a<20 \mathrm{AU}$.

The distribution of orbital separations and mass ratios in multiple systems can be compared to numerical simulations of star formation. Individual binary star systems that eclipse, or that can be spatially resolved, can be used to make precise measurements of fundamental physical properties of stars, such as mass and radius (see Southworth et al. 2011 and Torres et al. 2012 for recent examples). These measurements provide primary observational constraints on theoretical models of stellar structure and evolution. At the bottom of the stellar 
main sequence only a handful of these systems are known, and measurements deviate from theoretical expectations at the level of a few percent (Chabrier et al. 2007; Torres 2013; Feiden \& Chaboyer 2012; Terrien et al. 2012a). For this reason, it is particularly important to identify bright low-mass binaries over a wide range of orbital periods.

Previous large spectroscopic surveys designed to study M-dwarf stellar properties (e.g., Reiners et al. 2012; Jenkins et al. 2009) have included $\sim 300 \mathrm{M}$ dwarfs. In this paper, we describe an ongoing, extensive M-dwarf spectroscopic survey of $1404 \mathrm{M}$ dwarfs designed to detect low-mass companions, quantify the statistics of stellar multiplicity, and measure basic stellar physical parameters at the bottom of the main sequence. Using 253 of the 1404 stars that have been observed so far, we illustrate the techniques employed to measure observational and model-derived stellar parameters, and we demonstrate the concurrent use of RV monitoring and AO imaging to search for stellar companions to our sample of $\mathrm{M}$ dwarfs. The reduced and calibrated spectra for this first set of stars will be publicly available as part of the SDSS-III DR10 data release (Ahn et al. 2013) in summer 2013 and spectra for all stars will be released in 2014 December as part of the SDSS-III final data release. In addition to providing spectroscopic rotational velocities and radial velocities, this paper serves as an introduction to this unparalleled data set, lays out our target selection choices, and highlights some of the science investigations we are undertaking.

\section{THE APOGEE M-DWARF SURVEY}

The APOGEE (Majewski et al. 2010) is a high-resolution ( $R \sim 22,500)$, NIR ( $H$ band), multi-object, fiber-fed, and cryogenically cooled spectrograph (Wilson et al. 2010, 2012). The instrument is part of SDSS-III (Eisenstein et al. 2011), attached to the $2.5 \mathrm{~m}$ SDSS telescope (Gunn et al. 2006) at Apache Point Observatory, and covers a wide field of view ( $3^{\circ}$ diameter). The instrument can observe up to 300 targets simultaneously on a three-segment mosaic of Teledyne H2RG $2048 \times 2048$ detector arrays. Each detector has a wavelength range of $\sim 0.07 \mu \mathrm{m}$ and covers $1.514-1.581 \mu \mathrm{m}$ (blue), 1.586-1.643 $\mu \mathrm{m}$ (green), and 1.643-1.696 $\mu \mathrm{m}$ (red), respectively. The entire assembly is enclosed in a vacuum shell and is intrinsically very stable.

Our M-dwarf survey is an ancillary program to the main SDSS-III APOGEE survey, which began in 2011 September and will end in 2014 June. The observations are made during bright time. The light from the telescope is focused onto standard SDSS plugplates which have holes drilled in them to accommodate fibers. A total of $300120 \mu \mathrm{m}$ fibers are attached to the plugplate. Each fiber has a $2^{\prime \prime}$ diameter. The 300 fibers on each plate are allocated as follows: 230 are placed on science targets, 35 on telluric standards (hot stars used to calibrate and remove telluric features), and another 35 fibers are placed in sky regions devoid of celestial objects in order to obtain sky spectra (D. Nidever et al., in preparation). Multiple visits are made to many of the APOGEE fields during the course of the survey, allowing us to monitor RV variability among our sample of stars.

The main goal of APOGEE is to measure radial velocities and chemical abundances of $10^{5}$ red giant branch stars spanning various Galactic environments such as the bulge, disk, bar, and halos (S. R. Majewski et al., in preparation). Hence, the SDSS-III survey footprint primarily spans these regions. A detailed discussion of the APOGEE target selection and field plan can be found in Zasowski et al. (2013). Targets from our survey were generally distributed among fields with 3-24 visits, though some M-dwarf targets are also present on single-visit fields. The $M$ dwarfs can be identified in the DR10 release data (Ahn et al. 2013) by looking for bit flag 19. Spectra with this bit flag correspond to this program.

The APOGEE data reduction process is described in detail in D. Nidever et al. (in preparation). Products from the data reduction pipelines include: (1) apCframe files containing individual one-dimensional, dithered, wavelength calibrated spectra that represent individual exposures taken over the course of a single visit; (2) apVisit files consisting of co-added apCframe spectra from a single visit that are wavelength calibrated, flux normalized, and telluric corrected; and (3) apStar files including RV-shifted, co-added apVisit spectra and a best matching synthetic spectrum. A detailed list of various file formats are given in D. Nidever et al. (in preparation) and also described in detail as part of the SDSS-III DR10 release.

The goal of this survey is to characterize $M$ dwarfs through measurement of projected rotational velocities, absolute RV, and metallicity, and to discover new low-mass companions. This multi-epoch spectroscopic survey is sensitive enough to detect low-mass stellar and sub-stellar companions to $\mathrm{M}$ dwarfs and will also shed light on the statistics of stellar multiplicity at the bottom of the main sequence. Furthermore, these observations will create an atlas of high-resolution spectra in the NIR covering a wide range of sub-spectral types. These spectra are critical for modeling chemical abundances and probing the physical processes that occur in the complex atmospheres of $\mathrm{M}$ dwarfs. As an ancillary project to the APOGEE survey, this project has been awarded 6000 fiber hours ${ }^{22}$ over the SDSS-III survey.

\subsection{Target Selection}

Targets for the APOGEE M-dwarf survey primarily consist of stars from the all-sky Lepine and Shara proper motion North catalog (Lépine \& Shara 2005, LSPM-N hereafter) and the allsky catalog of bright M dwarfs (Lépine \& Gaidos 2011, LG11 hereafter).

The LSPM-N catalog contains stars with proper motions $\mu>$

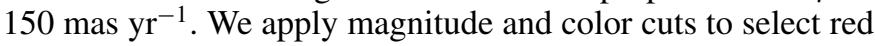
stars that fall within the APOGEE target field plan $(7<H<12$; $\left.V-K>5.0 ; 0.4<J-H<0.65 ; 0.1<H-K_{\mathrm{s}}<0.42\right)$. Contamination from $\mathrm{M}$ giants in a sample of $\mathrm{M}$ dwarfs is not uncommon, but they can be distinguished from dwarfs using infrared color cuts. Stars that pass these selection criteria and lie on planned APOGEE main survey fields are selected as targets. Aspects of this selection are also discussed in Zasowski et al. (2013), which describes target selection for the entire APOGEE survey, but are presented in more detail here.

The LG11 catalog became available only after we had submitted targets for initial plate drilling. LG11 is an end result of a careful application of infrared color and magnitude cuts to stars in the SUPERBLINK proper motion survey with the goal of selecting bright $\mathrm{M}$ dwarfs. Stars in the catalog are limited

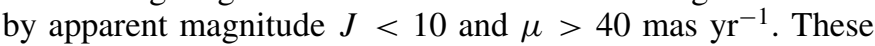
magnitude, color, and proper motion cuts enable the selection of bright $\mathrm{M}$ dwarfs while weeding out giants in the sample. When the catalog became available we used it to select targets for future plate drillings. To comply with our observational constraints, we apply declination $\left(\delta>0^{\circ}\right)$ and magnitude cuts to their sample ( $7<H$ to avoid saturating the APOGEE detector array). An important point to emphasize is that while our LSPM-N cuts were designed to select stars of spectral type M4 and later, our

\footnotetext{
22 One fiber hour is an hour-long exposure of a single target.
} 


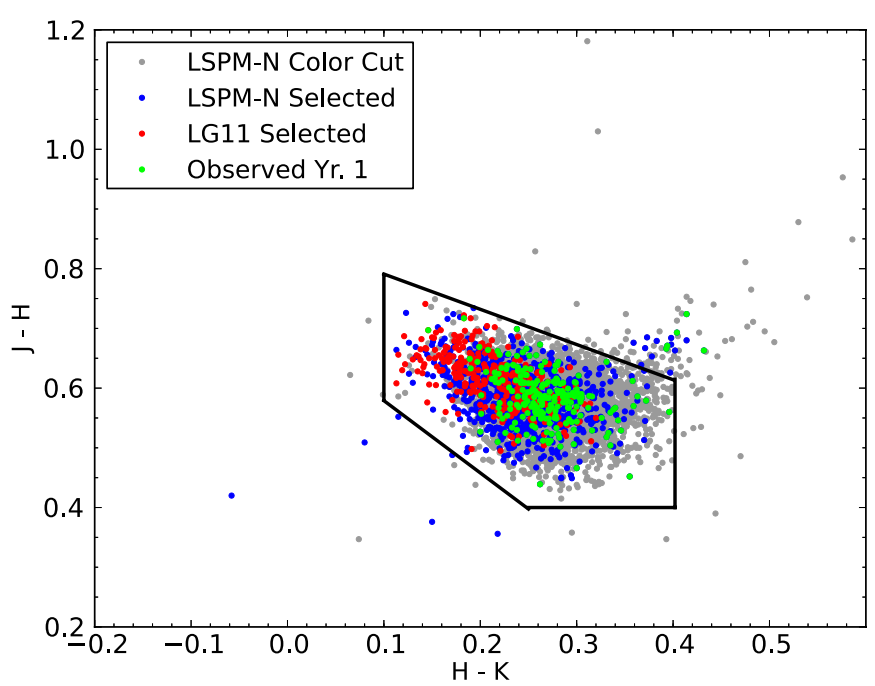

Figure 1. Infrared color-color diagram of our sample. The gray points are the 7059 LSPM-N stars obtained using the magnitude and color-cuts described in Section 2.1. The LSPM-N (blue), LG11(red), and stars observed in year 1 (green) are also plotted. Stars within the box, defined as the "red dwarf box" in LG11, are likely to be dwarfs, though some contamination by giants is possible. (A color version of this figure is available in the online journal.)

LG11 selection includes M dwarfs of all spectral types. The target selection in color-color space is illustrated in Figure 1. The region defined by Equations (9)-(13) from LG11 should contain stars that are potentially red dwarfs, while those objects outside the box, especially the reddest objects, are more likely $\mathrm{M}$ giants. We find that the majority of our target stars selected from LSPM-N (blue points) and LG11 (red points) lie within this box in color-color space.

Each APOGEE field consists of a circular plate with a radius of 1.49 . The plate has a bolt at the center with a radius of $5^{\prime}$ that cannot accept fibers. We assembled our final target list by cross-correlating our master target list with the area covered by each APOGEE plate. We anticipate observations of more than $1400 \mathrm{M}$ dwarfs over the course of the survey.

In the first year of the SDSS-III APOGEE survey 285 of these stars were submitted during plate drilling, of which 253 were observed (the remaining 32 were lost due to fiber conflicts with other targets or fell off the inner or outer plate edge when plate centers were slightly adjusted during final design). Figure 2 shows our entire sample in terms of color, magnitude, and approximate spectral types. The LSPM-N and LG11 (gray) are our total sample. The 253 targets observed during the first year are plotted as red filled circles (LSPM-N) and green filled triangles (LG11). As a result of the timing of the LG11 catalog release, most of stars observed during the first year were selected from LSPM-N and have spectral types of M3.5 and later. The observations of these stars have produced 1127 spectra. The distribution of their $H$ magnitude is shown in Figure 3. The 2MASS IDs of the $253 \mathrm{M}$ dwarfs observed during the first year along with their $J, H$, and $K$ magnitudes are listed in Table 1 .

We have also deliberately targeted some calibration stars, which include RV standards from the California Planet Survey, $v \sin i$ standards from literature (Jenkins et al. 2009), stars from the MEarth Project (Nutzman \& Charbonneau 2008), and $\mathrm{M}$ dwarfs in the Kepler field that are known to be active (Ciardi et al. 2011; Walkowicz et al. 2011). The selection of calibrators do not follow the stringent magnitude and color-cuts used as listed above, with the exception of bright magnitude cut:

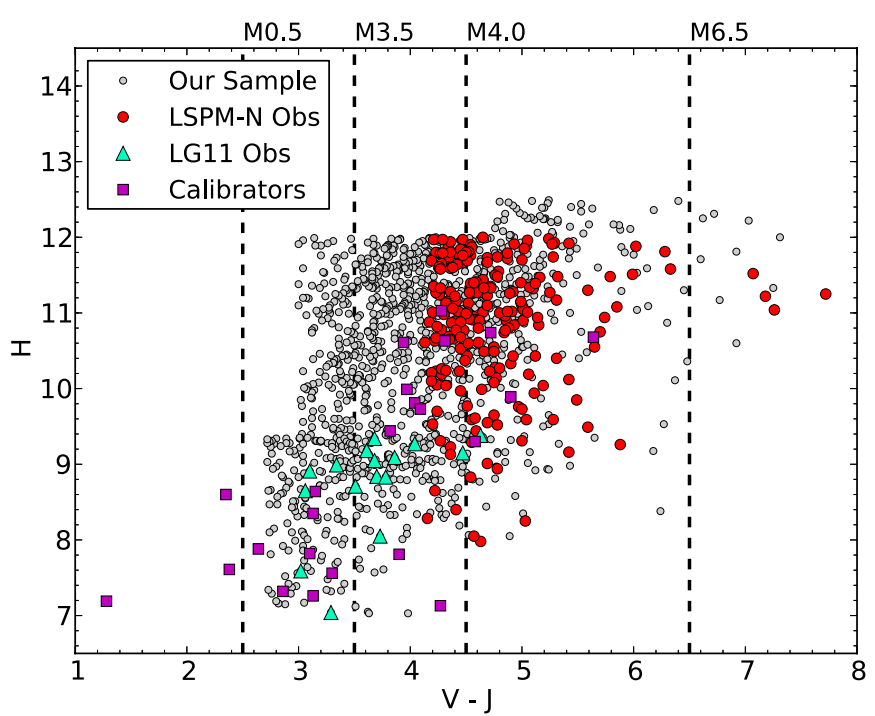

Figure 2. Entire APOGEE M-dwarf sample (gray filled circles) and calibrators (purple filled squares). The stars observed in the first year (2011 September-2012 July) are shown in color: LSPM-N (red filled circles), LG11 (green filled triangles). The vertical dash lines mark approximate positions of M-dwarf spectral sub-types.

(A color version of this figure is available in the online journal.)

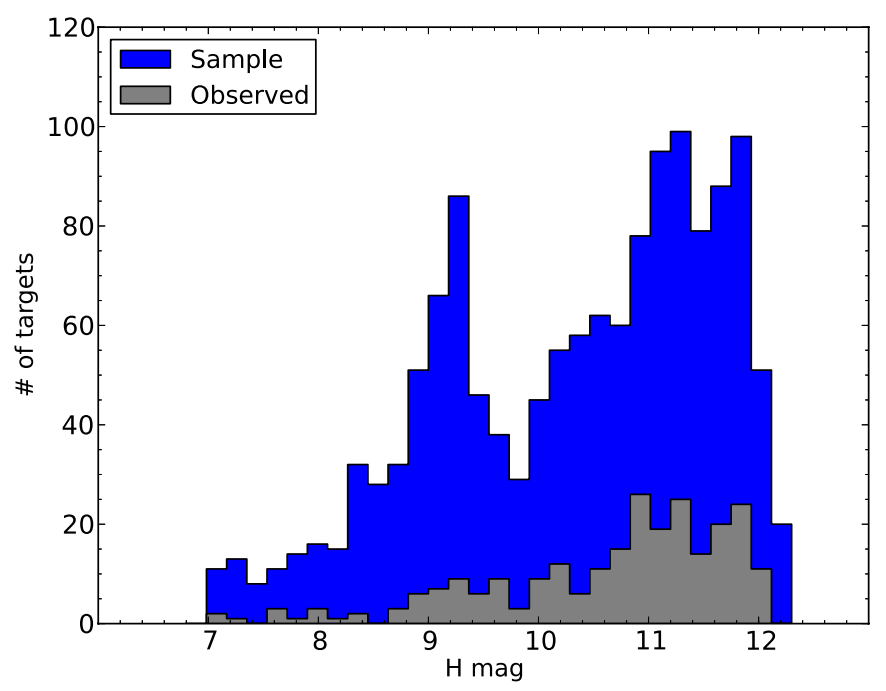

Figure 3. Distribution of $H$ magnitudes of the targets observed during the first year (gray; 2011 September-2012 July) and our entire sample (blue).

(A color version of this figure is available in the online journal.)

$H>7.0$. Figure 2 shows calibrators in our sample (green filled squares). The observed calibrators are listed in Table 2. In total, 25 calibration stars have been observed in the first year.

In order to explore the relationship between $\mathrm{M}$ dwarf subtype and features in the APOGEE high-resolution spectra, we constructed a set of spectral templates, using the combined spectra in the apStar files. For each subtype, we grouped the spectra by their $V-J$ color, which we calibrated to spectral type using the relation from Lépine \& Gaidos (2011) and bins centered on integer spectral types (e.g., M3 contains all stars with $V-J$ colors corresponding to M2.5 to M3.5). It is important to note that the $V$ magnitudes for many of these targets are based on scans of photographic plates and are therefore only reliable to $\pm 0.5 \mathrm{mag}$. This error in $V$ corresponds to approximately 1-2 subtypes. We then shifted these spectra to a common RV and interpolated to a common wavelength grid. The strong remaining sky lines and other artifacts are removed by first interpolating over pixels in 
Table 1

Targets Observed During the First Year of the APOGEE M-Dwarf Survey

\begin{tabular}{|c|c|c|c|c|c|c|c|c|c|c|}
\hline $\begin{array}{l}\text { Object } \\
\text { (2MASS ID) }\end{array}$ & $\begin{array}{c}J \\
(\mathrm{mag})\end{array}$ & $\begin{array}{c}H \\
(\mathrm{mag})\end{array}$ & $\begin{array}{c}K \\
(\mathrm{mag})\end{array}$ & $\begin{array}{c}\text { Visits } \\
\text { Planned }\end{array}$ & $\begin{array}{l}\text { Visits } \\
\text { Year } 1\end{array}$ & $\begin{array}{c}\mathrm{RV} \\
\left(\mathrm{km} \mathrm{s}^{-1}\right)\end{array}$ & $\begin{array}{c}\sigma-\mathrm{RV} \\
\left(\mathrm{km} \mathrm{s}^{-1}\right)\end{array}$ & $\begin{array}{c}v \sin i \\
\left(\mathrm{~km} \mathrm{~s}^{-1}\right)\end{array}$ & $\begin{array}{c}\sigma-v \sin i \\
\left(\mathrm{~km} \mathrm{~s}^{-1}\right)\end{array}$ & Binary \\
\hline $2 \mathrm{M} 00034394+8606422$ & 12.307 & 11.738 & 11.485 & 5 & 3 & 10.80 & 0.28 & 13.20 & 1.50 & $\ldots$ \\
\hline $2 \mathrm{M} 00085424+6716518$ & 11.674 & 11.131 & 10.836 & 5 & 12 & 10.07 & 0.19 & 27.60 & 1.40 & $\ldots$ \\
\hline $2 \mathrm{M} 00110613+7202521$ & 12.247 & 11.660 & 11.414 & 4 & 12 & -7.64 & 0.24 & 4.20 & 1.70 & $\ldots$ \\
\hline 2M00131578+6919372 & 8.556 & 7.984 & 7.746 & 4 & 12 & 13.57 & 0.17 & 6.00 & 0.20 & $\ldots$ \\
\hline $2 \mathrm{M} 00184520+7040399$ & 12.185 & 11.620 & 11.389 & 4 & 12 & 0.05 & 0.15 & $<4.00$ & $\ldots$ & $\ldots$ \\
\hline $2 \mathrm{M} 00185999+5836527$ & 11.839 & 11.264 & 11.009 & 6 & 12 & -7.75 & 0.38 & $<4.00$ & $\ldots$ & $\ldots$ \\
\hline 2M00222083+8619567 & 11.564 & 11.018 & 10.675 & 5 & 3 & -36.70 & 0.10 & 8.10 & 0.60 & $\ldots$ \\
\hline $2 \mathrm{M} 00234573+5353478$ & 12.060 & 11.483 & 11.179 & 3 & 12 & 5.24 & 0.47 & 10.10 & 1.20 & $\ldots$ \\
\hline $2 \mathrm{M} 00251480+6225017$ & 12.306 & 11.777 & 11.461 & 6 & 12 & 29.55 & 0.26 & 6.90 & 1.60 & $\ldots$ \\
\hline $2 \mathrm{M} 00251602+5422547$ & 11.775 & 11.215 & 10.819 & 3 & 12 & -63.27 & 0.20 & 9.20 & 0.40 & $\ldots$ \\
\hline
\end{tabular}

Note. The table includes infrared magnitudes, absolute RVs, projected rotational velocities, and estimated errors on absolute RV and $v$ sin $i$.

(This table is available in its entirety in machine-readable and Virtual Observatory (VO) forms in the online journal. A portion is shown here for guidance regarding its form and content.)

the target spectrum that were seen to have significant flux in the sky spectrum, and then interpolated over remaining sharp (single-pixel) features that had significantly higher or lower values than neighboring regions of the spectrum. With these cleaned spectra, we constructed an unweighted average spectrum for each spectral type. The spectral type bins contained as few as three (M1) or four (M8) spectra and as many as 95 (M4) spectra. Finally, we constructed artificial spectra for each 0.1 subclass by linearly interpolating between subclasses for each pixel.

We then constructed a routine to apply these templates to estimate the spectral type for any APOGEE M-dwarf spectrum. We selected regions that showed the most sensitivity to spectral type (Figure 4), and set the remaining spectrum to a flat continuum. For each target we then performed a cross-correlation on these filtered spectra with the equivalently filtered template spectra. The 0.1 subclass with the highest cross-correlation value was taken to be the estimated spectral type. Although this method is imprecise, it can be efficiently applied to estimate spectral types for large sets of targets with poorly constrained visual magnitudes. And despite the high uncertainties in the $V-J$ colors, the likely range of $v \sin i$ values for our targets, and the possible multiplicity of many targets, these templates clearly demonstrate the regions of $\mathrm{M}$-dwarf spectra in the $H$ band that are the most sensitive to spectral type. However, the spectral type assigned for each template spectrum is not rigorous, as it is based only on the definition of each $V-J$ color bin. We therefore take the 1-2 subtype uncertainty in the mapping of $V-J$ to spectral type as the estimated uncertainty in the resulting spectral types. Bins with larger numbers of targets likely average out the effects of other stellar parameters such as $v \sin i$ and metallicity, but probing the specific effects of these parameters is beyond the scope of this paper.

In addition to the $\mathrm{M}$ dwarfs directly selected as part of our ancillary program, 300 other field M dwarfs have been serendipitously observed by APOGEE. We do not discuss those in this intermediate data release paper but they will be included in the final survey analysis.

\section{INITIAL RESULTS}

Our APOGEE M-dwarf spectra span a wide range of signalto-noise ratio $(\mathrm{S} / \mathrm{N})$. Depending on the target brightness and observing conditions, the $\mathrm{S} / \mathrm{N}$ of individual APOGEE visits varies from a few tens to several hundred per resolution element.
The number of visits available for each target for the stars observed in the first year of the survey also varies: some targets only have a single visit so far, while others have 12 or more. In the following subsections we present initial results based on the analysis of spectra from Year 1 of the survey.

\subsection{Projected Rotational Velocities: $v \sin i$}

We measured projected rotational velocities for all of our targets using cross-correlation techniques. To maximize the precision of our measurement, we use the entire APOGEE spectral range. The first method cross-correlates a rotationally broadened synthetic spectrum against an observed target spectrum, and derives $v \sin i$ by maximizing the amplitude of the resulting correlation peak. The second method cross-correlates the observed spectrum of a slowly rotating template star against the observed target spectrum and measures the FWHM of the resulting correlation peak. This width is compared to tabulated values obtained by rotationally broadening the template spectrum and cross-correlating it against the unbroadened version of itself. Both of these methods are described in further detail below.

\subsubsection{Projected Rotational Velocities: Method I}

Our first method for measuring the $v \sin i$ of a target star uses a synthetic template spectrum with stellar characteristics $\left(T_{\mathrm{eff}}\right.$, $\log g$, and $[\mathrm{M} / \mathrm{H}])$, spectral resolution, and wavelength sampling that match those of the target. The template is rotationally broadened over a wide range of $v \sin i$ to generate a suite of broadened synthetic templates. Each broadened template is cross-correlated against the target spectrum, and the amplitude of the resulting correlation peak is measured. The correlation amplitude varies slowly with the template $v \sin i$, and the peak of this function determines the best $v \sin i$ estimate. This technique utilizes the entire free-spectral range that we deem to be free of telluric absorption and night sky $\mathrm{OH}$ emission, and we have used it in several past analyses of cool star spectra (e.g., van Eyken et al. 2012; Bender \& Simon 2008). Observations with S/N or spectral resolution insufficient for more traditional approaches that examine the line profiles of specific individual spectral features (Gray 1992), or where the spectral line density implies significant line blending, can still utilize this correlation based approach.

Carrying out this analysis requires a priori knowledge of the target star's $T_{\text {eff }}, \log g$, and $[\mathrm{M} / \mathrm{H}]$, so an appropriate synthetic template can be selected. An inaccurate choice at this point can 

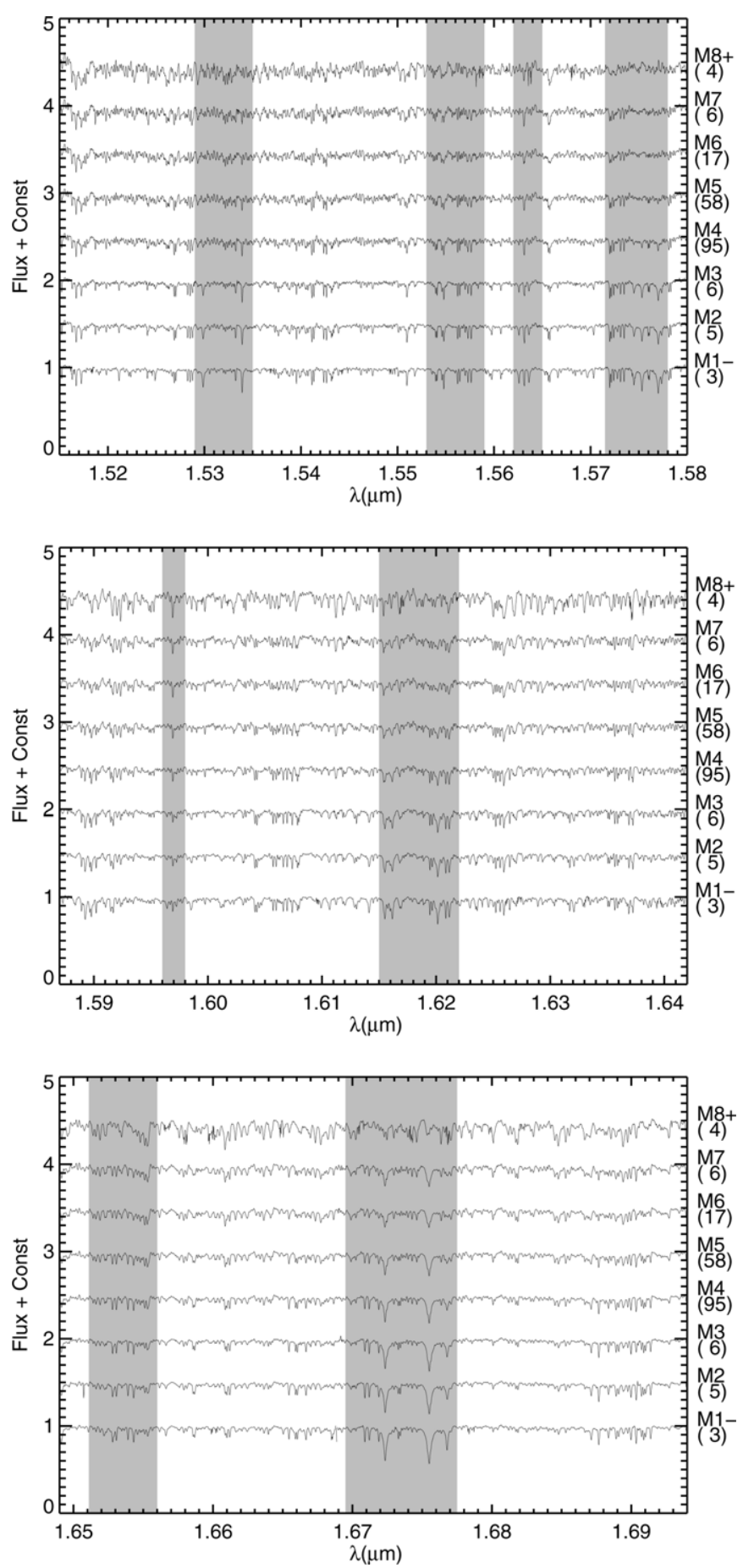

Figure 4. Average spectrum for each approximate spectral type, binned by $V-J$ color. The gray regions highlight the spectral-type sensitive regions that were used for spectral type estimation, and the numbers in parentheses indicate the number of targets in each bin. The three plots correspond to blue chip (top), green chip (center), and red chip (bottom).

introduce systematic errors in the derived rotational velocities. In the initial APOGEE data reduction pipeline, each apVisit spectrum is cross-correlated against a large grid of synthetic spectra as part of an initial spectroscopic characterization. The section of this grid that corresponds to $T_{\text {eff }}<4000 \mathrm{~K}$, and is relevant for our M-dwarf sample, is composed of synthetic spectra from the standard BT-Settl grid $^{23}$ (Allard

\footnotetext{
${ }^{23}$ http://phoenix.ens-lyon.fr/Grids/BT-Settl/AGSS2009/
}

et al. 1997). These initial correlation-based estimates return $T_{\text {eff }}$ to the nearest $100 \mathrm{~K}$, and $\log g$ and $[\mathrm{M} / \mathrm{H}]$ to the nearest 0.5 dex. Most APOGEE spectra are subsequently passed through the APOGEE spectra analysis pipeline (ASPCAP), which rederives these parameters with much finer precision. However, ASPCAP results are currently not regarded as reliable for cool stars with $T_{\text {eff }}<4000 \mathrm{~K}$. As such, we average the $T_{\text {eff }}, \log g$, and $[\mathrm{M} / \mathrm{H}]$ derived for each visit spectrum from the synthetic grid, and use these values to select the appropriate BT-Settl model (Allard et al. 1997) for our $v \sin i$ analysis.

The spectral resolution of a model must be degraded to match the resolution of the corresponding target spectrum. APOGEE's spectral resolution varies across the spectral range, and also depending on the fiber location on the focal plane (Wilson et al. 2012), but the line spread function (LSF) for each fiber is automatically parameterized by the reduction pipeline as a Gauss-Hermite polynomial and stored along with the individual visit spectra. APOGEE targets with multiple visits are not guaranteed to be carried out using the same spectrograph fiber, although in practice they often are. Targets with visits distributed over multiple fibers will result in visit spectra with slightly different LSFs; these effectively get averaged together when the data pipeline co-adds the individual visit spectra. For simplicity, we only consider a target's average LSF, and approximate it as a Gaussian to derive the corresponding effective spectral resolution. We then degrade the synthetic template spectrum to the derived resolution.

We rotationally broaden the synthetic templates using a four parameter non-linear limb-darkening model (Claret et al. 2012; Claret 2000; Gray 1992), with parameters appropriate for the characteristics of the stellar spectrum and the APOGEE $H$-band bandpass, over a range of $v \sin i$ from $3 \mathrm{~km} \mathrm{~s}^{-1}-100 \mathrm{~km} \mathrm{~s}^{-1}$. Finally, we resample both the APOGEE spectra and synthetic models to log-lambda wavelength space (Tonry \& Davis 1979) in preparation for cross-correlation. The apStar files produced by the APOGEE data pipeline contain co-added spectra for each multi-visit target using two different co-adding schemes. Our $v \sin i$ analysis measures both co-added apStar spectra, and also each of the individual apVisit spectra. The $v \sin i$ from the individual visit spectra are then combined using a weighted average, with the weights derived from the $\mathrm{S} / \mathrm{N}$ of each visit. We take the standard deviation of the distribution as the measurement precision on $v \sin i$, and impose the following additional rules: (1) single visit spectra have default precision of $2 \mathrm{~km} \mathrm{~s}^{-1}$ and (2) multi-visit spectra with three or fewer visits have a minimum precision of $1 \mathrm{~km} \mathrm{~s}^{-1}$. In addition, we set a conservative floor in our ability to measure $v \sin i$ at $4 \mathrm{~km} \mathrm{~s}^{-1}$, which also corresponds to the minimum $v \sin i$ where the broadening kernel is resolved at APOGEE resolution and sampling. We tested template mismatch and resulting error propagation for few stars from our sample. This was done by varying $T_{\text {eff }}$ and $\log g$ by $200 \mathrm{~K}$ and $0.5 \mathrm{dex}$, respectively, from the APOGEE derived stellar parameters. We found these measured $v \sin i$ to be within the errors of $v \sin i$ measurements computed using APOGEE pipeline derived $T_{\text {eff }}$ and $\log g$. Furthermore, Method 1 was able to identify two cool dwarfs whose effective temperatures were erroneously computed by the APOGEE pipeline to be a few $1000 \mathrm{~K}$ due to low $\mathrm{S} / \mathrm{N}$ in their spectra. We further tested the method by measuring $v \sin i$ of main sequence stars in the main APOGEE survey. We found nine stars with spectral types of A5-K0 with a large range of previously reported $v \sin i$ values (Randich et al. 1996; Mermilliod et al. 2008). Figure 5 


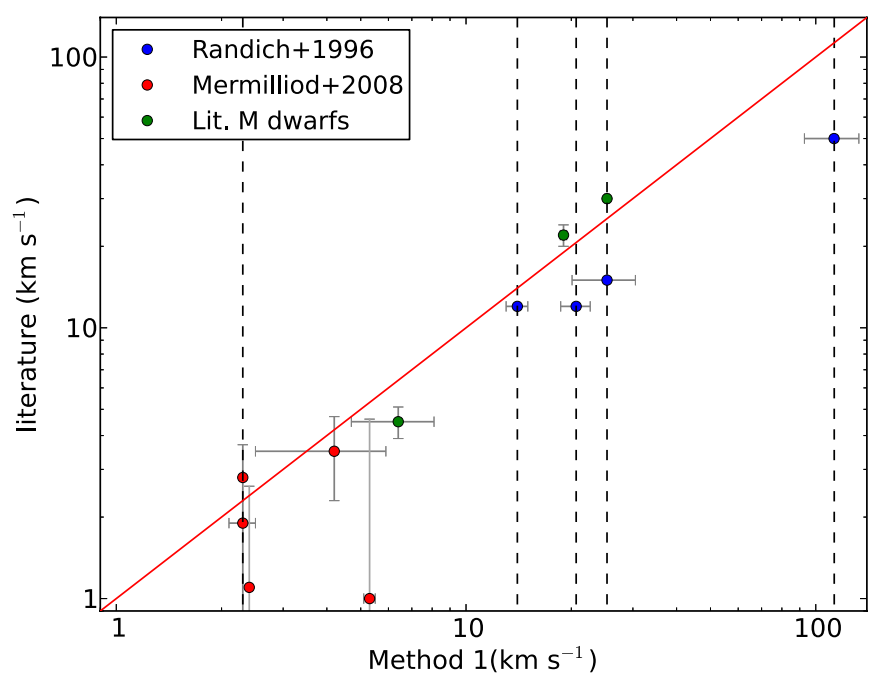

Figure 5. Comparison of literature $v \sin i$ measurements of stars with Method 1 . Stars in this figure include spectral types of A5 to M6. Literature M dwarfs are listed in Table 2. The solid line indicates 1:1. Literature stars that do not have reported errors are marked by dashed lines.

(A color version of this figure is available in the online journal.)

illustrates this effort. We find that $75 \%$ of our stars are within $1 \sigma$ of the $1: 1$ line.

\subsubsection{Projected Rotational Velocities: Method II}

Our second method measures $v \sin i$ by cross-correlating object spectra against a slowly rotating template spectrum of similar spectral type and measuring the FWHM of the crosscorrelation peak. This process is well established in the literature (e.g., Bailer-Jones 2004) and assumes that the line profile is primarily dominated by rotation.

Four slowly rotating stars are used as templates: 2M05470907-0512106 with $v \sin i=4.5 \mathrm{~km} \mathrm{~s}^{-1}$ (Jenkins et al. 2009) and 2M19125504+423937, 2M19121128+4316106, and $2 \mathrm{M} 19332454+4515045$ with rotation periods of 48.5, 59.0, and 42.6 days, respectively, determined from Kepler photometry (McQuillan et al. 2013). We used the apVisit and apStar files in the analysis.

Prior to cross-correlation, the spectra are processed to remove regions of poorly corrected telluric lines or residual night sky emission. The former are generally identified by negative or non-number values in the spectrum. To identify the latter, the continuum level is estimated and regions 5\% larger than the continuum are considered probable emission features. Once identified, these features are removed by interpolating over them using the good parts of the spectrum. Next, the spectra for each chip are normalized by the median. Additionally, a few very strong atomic lines were masked out. Although such lines are good for RV centering, they tend to have significant wings, leading to less Gaussian-like profiles. These regions are: $15630.5-15633.5,16110.5-16113.5,16719-16729$, and 16748.5-16763.5 $\AA$, in the stellar rest frame and were identified by eye. In some cases the automated APOGEE reduction pipeline set fluxes to zero. Such regions are masked prior to analysis. In addition, the blue chip was ultimately not used in the analysis for reasons described below.

To map FWHM to $v \sin i$, we broaden each of the template spectra with a rotation kernel for a range of $v \sin i$ between $2-30 \mathrm{~km} \mathrm{~s}^{-1}$ in increments of $2 \mathrm{~km} \mathrm{~s}^{-1}$, from $30-60 \mathrm{~km} \mathrm{~s}^{-1}$ in increments of $5 \mathrm{~km} \mathrm{~s}^{-1}$, and from $60-90 \mathrm{~km} \mathrm{~s}^{-1}$ in increments of $10 \mathrm{~km} \mathrm{~s}^{-1}$. The rotational kernel is given by Equation (17.12) of Gray (1992), using a limb darkening parameter $(\epsilon)$ of 0.25 , which is appropriate for the NIR $H$ band (Claret et al. 2012). These broadened templates are used as object spectra and run through the same pipeline as the program stars. For a given kernel $v \sin i$ we averaged the resulting FWHM of all the broadened spectra for each combination of chip and un-broadened template. This allowed us to create a mapping from FWHM to $v \sin i$ for each template and chip. The true $v \sin i$ modeled by the artificially broadened template includes both the intrinsic rotation of the template stars and the applied rotation kernel. To account for the intrinsic rotation, we used $4.5 \mathrm{~km} \mathrm{~s}^{-1}$ as an estimate of the intrinsic stellar rotation, which was measured for one of the templates in the literature. It is adopted for the remaining templates on the basis that the FWHM of the object spectra do not appear to be systematically higher or lower for any of the templates. We then set the $v \sin i$ of the artificially broadened spectra to be the quadrature sum of the intrinsic $v \sin i$ and the kernel $v \sin i$. To apply this mapping to the object spectra, we fit a sixth-order polynomial to the relationships between measured FWHM and total $v \sin i$ (the combination of the intrinsic stellar rotation and kernel $v \sin i$ ), with FWHM as the independent variable. In addition, the relationship for the blue chip was very different from that of the red and green chips, and we decided to exclude that chip in this paper.

Before using the polynomials to map the FWHM of the object spectra to $v \sin i$, we first apply a quality cut requiring that the height of the cross-correlation peak be at least 0.4 and that the center of the cross-correlation peak be within $\pm 5 \mathrm{~km} \mathrm{~s}^{-1}$ of zero, since all apStar spectra should be in the stellar rest frame. These cuts should remove correlations for which the largest peak is in fact a noise peak. The FWHM measurements making this cut are mapped to $v \sin i$ using the polynomial fits. The upper or lower limits to $v \sin i$ are computed using the average FWHM plus or minus the standard deviation in the FWHM. These are used for upper and lower errors in $v \sin i$. In all of these cases, small FWHM that would otherwise map to negative values are set to zero. Next, we average together all the $v \sin i$ measurements for a given chip. We propagate the high and low $v \sin i$ errors by taking the quadrature sum of each. We also compute the standard deviation in the $v \sin i$ values $\left(\sigma_{\text {chip }}\right)$ as a measure of systematic errors arising from the different templates. Finally, we take a weighted average of the two individual chip $v \sin i$ 's to compute the final $v \sin i$, denoted $(v \sin i)_{f}$. The weights are given by $w=1 / \sigma_{\text {chip }}^{2}$. We set a floor of $\sigma_{\text {chip }}$ to $0.5 \mathrm{~km} \mathrm{~s}^{-1}$, to avoid giving overly high weights. The final error in the $v \sin i$ is given by the quadrature sum of the formal errors and the measured standard deviation.

\subsubsection{Projected Rotational Velocities: Comparing Method I and II}

The two methods, as discussed above, independently measure $v \sin i$ of the $\mathrm{M}$ dwarfs from Year 1, and are directly compared in Figure 6. For most stars with $v \sin i>9 \mathrm{~km} \mathrm{~s}^{-1}$, the measurements derived from two methods are within $2 \sigma$ of the 1:1 line. However, for $v \sin i$ below $9 \mathrm{~km} \mathrm{~s}^{-1}$ we find that Method 2 either underestimates velocities or Method 1 overestimates them for some of the stars. Nonetheless, $\sim 75 \%$ the slow rotators are within $1 \sigma$ of the $1: 1$ line.

Both methods rely on a library of template spectra. Method 1 uses the BT-Settl models (Allard et al. 1997) at the finest grid these atmospheric models provide. However, Method 2 is limited by a small number of observed templates. This is a 


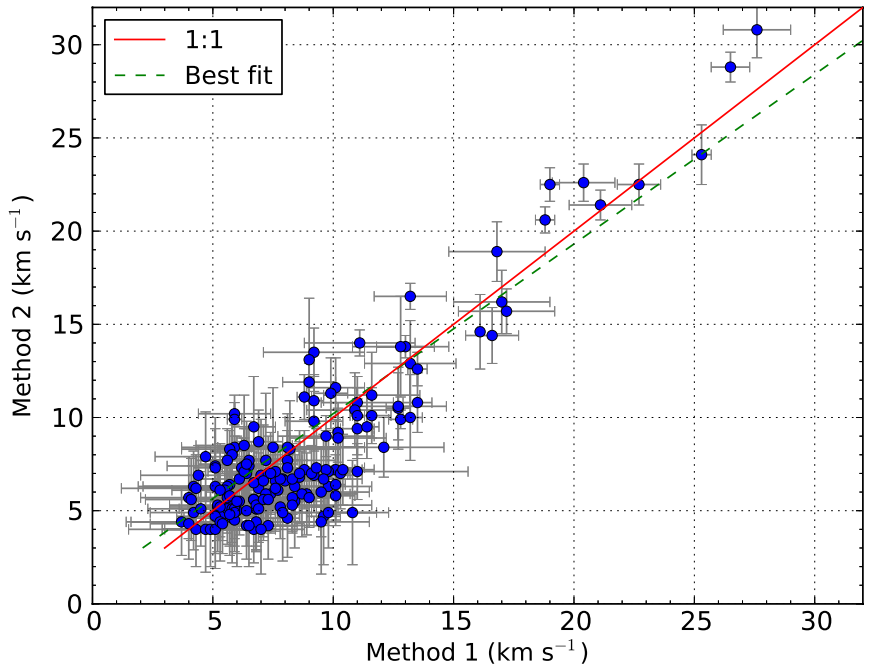

Figure 6. Comparison of $v \sin i$ measurements of our M-dwarf sample using Method 1 and Method 2, as described in Section 3.1. The solid line represents perfect agreement. The linear regression best fit $(y=B x+A, B=1.099$, $A=-1.212$ ) is indicated by a dashed green line.

(A color version of this figure is available in the online journal.)

consequence of finding few $v \sin i$ templates that overlapped with pre-designed APOGEE fields. The preliminary APOGEE spectra pipeline analysis suggests that all of the observed templates have $T_{\text {eff }}$ between $3300-3500 \mathrm{~K}$ while their $V-J$ colors confirm them to be early-M dwarfs. The same analysis done on our sample indicates a $T_{\text {eff }}$ range of $3500 \mathrm{~K}-2700 \mathrm{~K}$ with the mean $T_{\text {eff }}$ of $3300 \mathrm{~K}$. However, there are $\sim 70$ stars with $T_{\text {eff }}$ below $3100 \mathrm{~K}$. As the chemical composition of $\mathrm{M}$ dwarfs changes faster than the effective temperature (Reiners et al. 2012), early-M-dwarf templates are likely to give erroneous measurements for late-M dwarfs.

We report $v \sin i$ measurements as derived by Method 1 . These measurements are listed in Table 1, along with their uncertainties. Figure 7 plots $v \sin i$ measurements derived from Method 1 (blue filled circles) and the literature (gray filled circles) as a function of $V-J$ color. Upper limits on slow rotators of $4 \mathrm{~km} \mathrm{~s}^{-1}$ from this work are indicated by downward arrows.

Our color and magnitude cuts for the Year 1 sample selected dwarfs with spectral types M3-M7. However, a large portion of our observed sample have spectral types between M3.5 and M5.0 as seen in Figure 7. Photometric (Irwin et al. 2011) and spectroscopic $v \sin i$ measurements of M dwarfs (e.g., Jenkins et al. 2009; Mohanty \& Basri 2003) find that M dwarfs with later spectral types take much longer to spin down as compared to their earlier counterparts. We find the same trend in our sample. For M dwarfs between M3.5 and M5.0, we see a spread in $v \sin i$ values from our measurement sensitivity threshold of $4 \mathrm{~km} \mathrm{~s}^{-1}$ to $10 \mathrm{~km} \mathrm{~s}^{-1}$ with very few over $10 \mathrm{~km} \mathrm{~s}^{-1}$. Analysis of over $1000 \mathrm{M}$ dwarfs from our second year, most of which are early-M dwarfs, will shed more light on the projected rotational velocity spread of early-M dwarfs.

\subsection{Relative Radial Velocities Using Telluric Modeling}

APOGEE is, in principle, very well suited for making precise RV measurements of low-mass stars because of the LSF stability intrinsic to a fiber-fed instrument housed in a vacuum enclosure. While the modest resolution of the instrument precludes precision at the level achieved by instruments designed specifically for RV planet searches at optical wavelengths, such as HARPS (Mayor et al. 2003), precision better

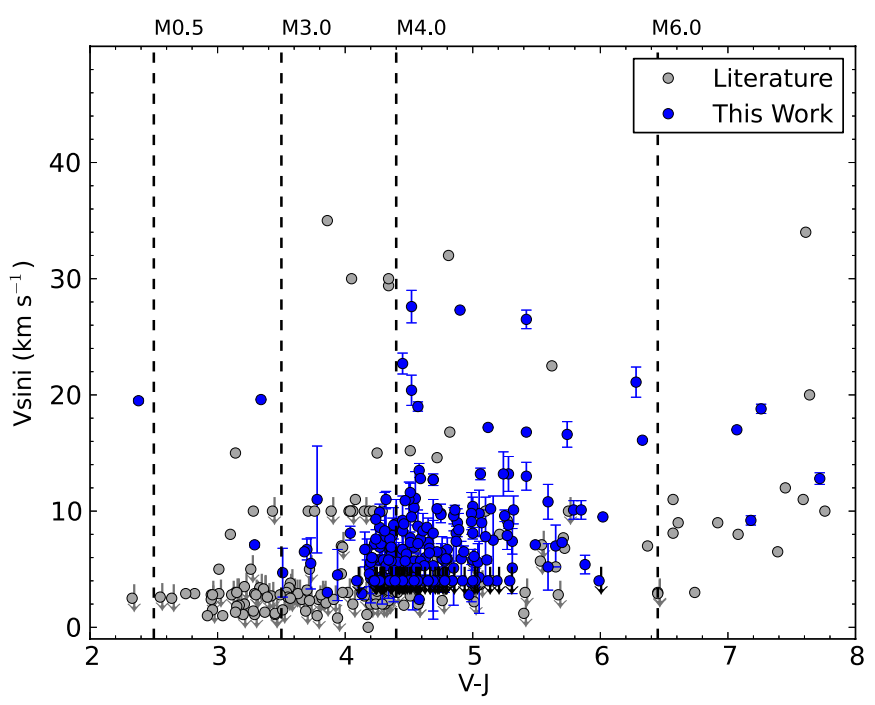

Figure 7. Measurements of $v \sin i$ for $\mathrm{M}$ dwarfs, including the measurements presented here (blue filled circles) and those from the literature (gray filled circles), for a range of $V-J$ colors. The dashed lines mark the approximate positions of M-dwarf spectral sub-types.

(A color version of this figure is available in the online journal.)

Table 2

Standard Stars Observed During the First Year of the APOGEE M-Dwarf Survey

\begin{tabular}{|c|c|c|c|}
\hline Star & Alt. Name & Standard Type & Reference \\
\hline $02085359+4926565$ & GJ3136 & $v \sin i$ & (a) \\
\hline $05030563+2122362$ & $\ldots$ & $v \sin i$ & (b) \\
\hline $05470907-0512106$ & GJ3366 & $v \sin i$ & (c) \\
\hline $14264827-0510400$ & HD126614 & RV & (d) \\
\hline $19051739+4507161$ & LHS 3429 & Activity & (e) \\
\hline $19121128+4316106$ & $\ldots$ & Activity & (e) \\
\hline $19125504+4239370$ & $\ldots$ & Activity & (e) \\
\hline $19185898+3812236$ & $\ldots$ & Activity & (f) \\
\hline $19235494+3834587$ & $\ldots$ & Activity & (f) \\
\hline $19241533+3638089$ & $\ldots$ & Activity & (f) \\
\hline $19265550+3844381$ & $\ldots$ & Activity & (f) \\
\hline $19271763+3913024$ & $\ldots$ & Activity & (f) \\
\hline $19294258+3733223$ & LHS6351 & Activity & (e) \\
\hline $19302029+3723437$ & $\ldots$ & Activity & (f) \\
\hline $19330692+4005066$ & $\cdots$ & Activity & (f) \\
\hline $19332454+4515045$ & $\ldots$ & Activity & (e) \\
\hline $19333940+3931372$ & $\ldots$ & Activity & (e) \\
\hline $19395886+3950530$ & $\ldots$ & Activity & (f) \\
\hline $19420033+4038302$ & $\ldots$ & Activity & (f) \\
\hline $19445931+4812415$ & $\ldots$ & Activity & (f) \\
\hline $19450736+3947341$ & $\ldots$ & Activity & (f) \\
\hline $19485718+5015245$ & $\ldots$ & Activity & (e) \\
\hline $19510930+4628598$ & GJ 1243 & $v \sin i$ & (b) \\
\hline $22392577+5719249$ & HIP 111854 & RV & (d) \\
\hline $23124910+4726557$ & LTT 16823 & RV & (d) \\
\hline
\end{tabular}

References. (a) Gizis et al. 2002; (b) Nutzman \& Charbonneau 2008; (c) Jenkins et al. 2009; (d) Chubak et al. 2012; (e) Ciardi et al. 2011; (f) Walkowicz et al. 2011.

than $50 \mathrm{~m} \mathrm{~s}^{-1}$ per epoch should be possible for targets brighter than $H=7.5$. Given the low masses of our targets, this is sufficient to detect a wide range of companions, including giant planets with short orbital periods.

Precise RV instruments generally rely on one of two strategies for calibrating the pixel-to-wavelength scale of the instrument and monitoring short- and long-term instrumental drifts: an emission line source coupled via an optical fiber to a 
stabilized instrument or an absorption reference gas cell in the telescope beam prior to the entrance of a slit spectrograph. The primary wavelength calibration of the APOGEE data relies on a combination of ThAr and UNe lamps (Redman et al. 2011, 2012), but the numerous telluric absorption features present in the APOGEE spectra can act as a wavelength reference, providing a secondary wavelength calibration that is obtained simultaneously with the stellar spectra. The bulk motion of the atmosphere causes instability in telluric absorption features at the 5-10 $\mathrm{m} \mathrm{s}^{-1}$ level (e.g., Figueira et al. 2010 and references therein). Given the resolution of APOGEE and the typical S/N of the spectra, we expect the RV precision limitations imposed by the intrinsic stability of the telluric lines to be a factor of a few smaller than the limitations imposed by the intrinsic information content of the spectra and the stability of the spectrograph.

To estimate small corrections to wavelength solutions derived from the emission line lamps alone, we forward model the APOGEE spectra using a technique similar to the one used in Blake et al. (2010) and Rodler et al. (2012), which are both based on the data analysis strategy outlined in Butler \& Marcy (1996). We model spectra spanning 850 pixels from the green chip, covering the approximate wavelength range 1.598 to $1.616 \mu \mathrm{m}$. While this is less than a third of the total spectral coverage of APOGEE, this region is rich with telluric and stellar spectral features and also suffers less from the undersampling of the LSF found at bluer wavelengths. Following Blake et al. (2010), we forward model the spectra as the product of a stellar template and telluric model convolved with the spectrograph LSF. For a stellar template, we use the apStar spectra, the weighted combination of all the individual spectra of each object, produced by the APOGEE pipeline. We deconvolve this apStar template with the LSF estimated by the APOGEE pipeline (D. Nidever et al., in preparation) using a Jansson technique based on that used in Butler \& Marcy (1996). Given enough APOGEE epochs over a wide enough range of barycentric velocity, this technique can effectively average out errors due to imprecise telluric modeling and residuals from the subtraction of bright sky lines. We note that constructing a template in this way makes all of our resulting RV measurements fundamentally differential and also relies on the assumption that any intrinsic stellar RV variations are small compared to the barycentric motions and the APOGEE pixels (1 pixel $\approx 5 \mathrm{~km} \mathrm{~s}^{-1}$ ).

In the spectral region we are focusing on, there are prominent telluric absorption features due to $\mathrm{CO}_{2}$. We calculate a highresolution telluric transmission model appropriate for average conditions at Apache Point Observatory using the radiative transfer code described in Blake \& Shaw (2011). Our model for the APOGEE spectra has four free parameters: one optical depth scale factor for the telluric model, two for a linear wavelength correction to the APOGEE pipeline wavelength solutions, and one for stellar velocity. We use the LSFs provided by the APOGEE pipeline. We fit the individual apCframe spectra, several of which are obtained at each epoch as part of a dither set. The model is first generated at a resolution seven times the APOGEE resolution, then interpolated to the APOGEE wavelength scale while explicitly integrating over the extent of each detector pixel. We use the AMOEBA downhill simplex method (Nelder \& Mead 1965) to fit the model to each apCframe spectrum and find the best-fit values for the four free parameters by minimizing $\chi^{2}$. Prior to fitting each spectrum we mask out regions known to have strong $\mathrm{OH}$ emission lines. Finally, we decorrelate the RV estimates from the forward modeling process against the measured skewness of the APOGEE pipeline LSFs

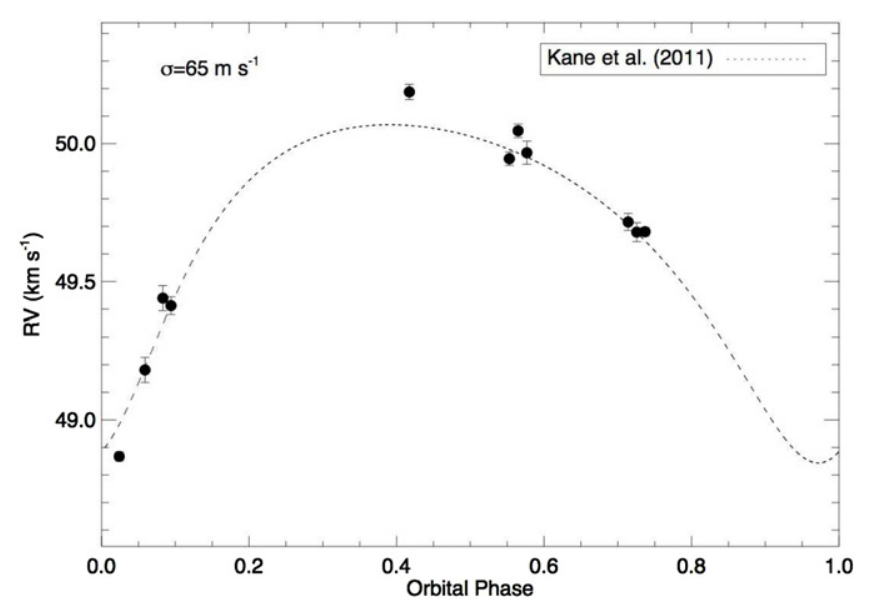

Figure 8. APOGEE relative RV measurements compared to the published orbit for HD114762. The $\sigma$ value indicates the standard deviation of the RV measurements after subtracting the Kane et al. (2011) orbit.

for each apCframe spectrum. This is done by fitting a linear relation between LSF skewness and RV, then subtracting a line with this best-fit slope from the RV estimates. We found that this correction improves the scatter of the resulting RV measurements by up to $30 \mathrm{~m} \mathrm{~s}^{-1}$.

In Figures 8 and 9 we show examples of RV precision, including a star known to be RV stable to better than $10 \mathrm{~m} \mathrm{~s}^{-1}$ and the giant planet companion to the star HD114762 (Latham et al. 1989; Kane et al. 2011). This was observed serendipitously by APOGEE as a telluric standard, and was independently recovered as a signal of a possible sub-stellar companion. It serves to demonstrate that NIR radial velocities even with a survey instrument are at the level of precision to enable discovery of giant planets. The telluric modeling techniques presented here are still being developed and improved, and more precise radial velocities derived from this analysis will be presented in future work.

\subsection{Barycentric Radial Velocities}

Barycentric radial velocities for each star are derived in the final step of the pipeline reduction process. The detailed description of this RV determination is given in D. Nidever et al. (in preparation). Here, we summarize the steps. RV is determined by cross-correlating a normalized observed spectrum with a library of synthetic BT-Settl models that span the effective temperature and $\log g$ range of $\mathrm{M}$ dwarfs with metallicities between -4.0 and 0.3 . First, a model template is selected through cross-correlating each model template from the library with the observed target. The best template is chosen through $\chi^{2}$ minimization. This template is then cross-correlated with the observed spectra of the target and velocity is estimated by fitting the cross-correlation function (CCF) with a Gaussian plus a linear fit. Finally, a barycentric correction is applied to this velocity measurement. The standard deviation of the RV measurements is $\sim 130 \mathrm{~m} \mathrm{~s}^{-1}$. The absolute RVs of stars from our sample are listed in Table 1. For the sake of consistency these velocities are the same as those released as part of the pipeline output with the DR10 release. The column of $\sigma-\mathrm{RV}$ in Table 1 shows the rms scatter in the measured radial velocities for stars that have measurements at three or more epochs.

Stars that show RV variation larger than $1 \mathrm{~km} \mathrm{~s}^{-1}$ or result in CCFs with distinct peaks are identified as potential binaries. These candidate binaries are listed in Table 4 along with the number of observations and their infrared magnitudes. Orbital 

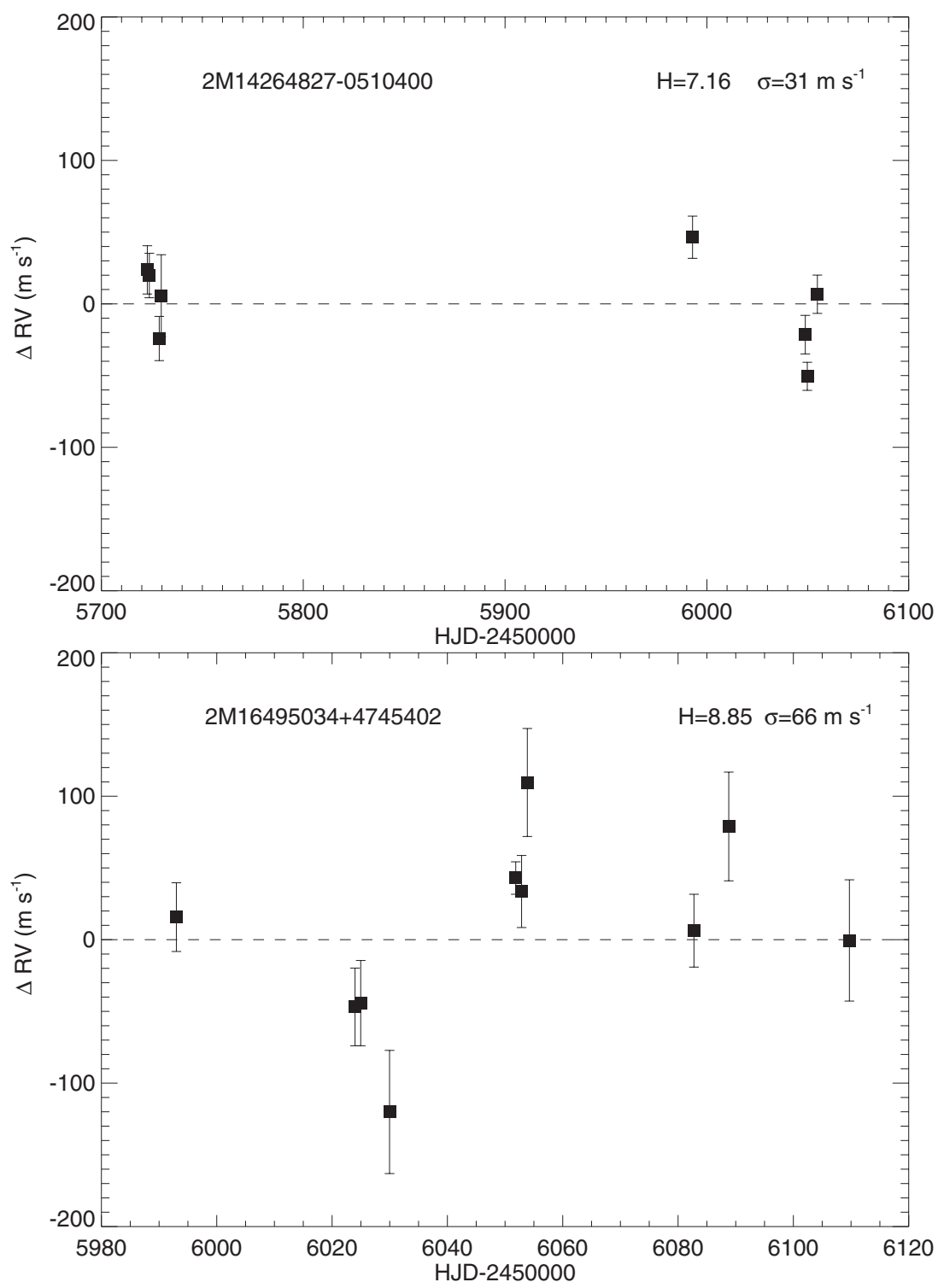

Figure 9. APOGEE relative RVs for two low-mass stars. 2M1426+0510 (top panel) is a K0 star with a known planetary companion, resulting in an RV semi-amplitude of $7.32 \mathrm{~m} \mathrm{~s}^{-1}$ at an orbital period of $3.41 \mathrm{yr}$ (Howard et al. 2010). 2M1649+4745 (bottom panel) has no known companion. The $\sigma$ values indicate the standard deviations of the RV measurements of the two stars.

parameters, mass ratios, and other analyses of these systems will be presented in separate papers, along with additional radial velocities and high-resolution spectra.

\subsection{Physical Parameters of M Dwarfs}

Important improvements in stellar atmospheric models of low-mass stars have resulted from the availability of new atomic line profile data and the inclusion of dust and clouds in the models. Atomic line profile data becomes especially important in situations where line blanketing and broadening are crucial, and stellar models incorporating these updated atomic data give a much improved representation of the details of the line shapes in optical and NIR spectra of cool dwarfs (Rajpurohit et al. 2012). The recent suite of synthetic BT-Settl models include dust and clouds in their computation of stellar atmospheres and therefore provide a good fit to the observed stellar spectrum. These model atmospheres are computed with the PHOENIX code assuming hydrostatic equilibrium and convection using mixing length theory (Prandtl 1926) with a mixing length of $1 / H_{\mathrm{p}}=2.0$ according to results of radiation hydrodynamics (Ludwig et al. 2006). The models are calculated using spherically symmetric radiative transfer, departure from LTE for all elements up to iron, the latest solar abundances by Asplund et al. (2009), Caffau et al. (2011), equilibrium chemistry, a database of the latest opacities and thermochemical data for atomic and molecular transitions, and monochromatic dust condensates and refractory indexes. Grains are assumed spherical and non-porous, and their Rayleigh and Mie reflective and absorptive properties are considered. The diffusive properties of grains are treated based on twodimensional radiation hydrodynamic simulations, including forsterite cloud formation to account for the feedback effects of cloud formation on the mixing properties of these atmospheres (Freytag et al. 2010).

We selected four APOGEE $M$ dwarfs that have also been observed by us with the $3 \mathrm{~m}$ telescope at the NASA Infrared Telescope Facility (IRTF) using the SpeX spectrograph, which provides wavelength coverage from $0.8-2.5 \mu \mathrm{m}$ at a 


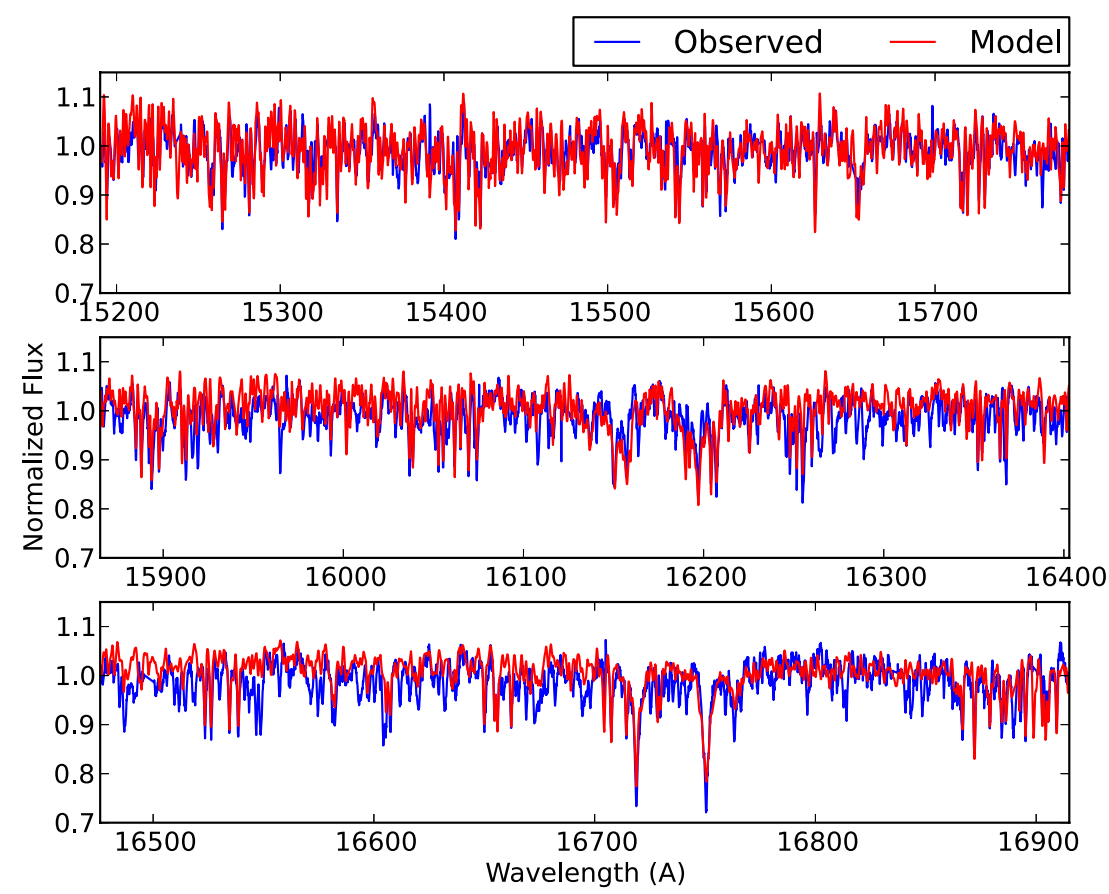

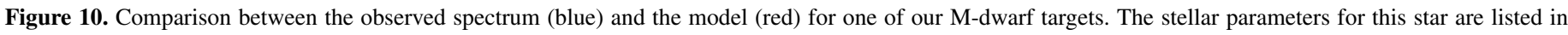

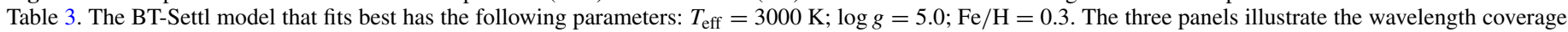
of three chips: the blue chip (top panel), the green chip (center panel), and the red chip (bottom panel).

(A color version of this figure is available in the online journal.)

Table 3

M-Dwarf Stellar Properties Derived from Model Fits

\begin{tabular}{|c|c|c|c|c|c|c|}
\hline $\begin{array}{l}\text { Star } \\
(2 \mathrm{MASS})\end{array}$ & $\begin{array}{c}{[\mathrm{M} / \mathrm{H}]} \\
\text { (Model) }\end{array}$ & $\begin{array}{c}{[\mathrm{M} / \mathrm{H}]} \\
(\text { Observed })^{\mathrm{a}}\end{array}$ & $\begin{array}{l}T_{\text {eff }} \\
(\mathrm{K})\end{array}$ & $\begin{array}{l}\log g \\
(\operatorname{cgs})\end{array}$ & $\begin{array}{l}T_{\text {eff }} \\
(\mathrm{K})^{\mathrm{b}}\end{array}$ & $\begin{array}{l}\log g \\
(\mathrm{cgs})^{\mathrm{b}}\end{array}$ \\
\hline $00251602+5422547$ & $0.0 \pm 0.25$ & $\ldots$ & $2700 \pm 100$ & 5.0 & 2700 & 4.0 \\
\hline $04125880+5236421$ & $0.3 \pm 0.26$ & $-0.04 \pm 0.12$ & $3000 \pm 100$ & 5.0 & 3300 & 5.0 \\
\hline $13451104+2852012$ & $0.3 \pm 0.20$ & $-0.13 \pm 0.12$ & $3200 \pm 100$ & 5.0 & 3300 & 5.0 \\
\hline $19081153+2839105$ & $0.0 \pm 0.20$ & $0.22 \pm 0.12$ & $3200 \pm 100$ & 5.0 & 3300 & 5.0 \\
\hline
\end{tabular}

Notes.

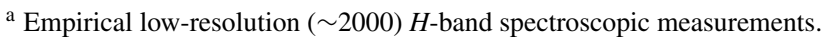

b Automated APOGEE pipeline values.

resolution of 2000. These targets are part of an ongoing program to derive empirical $\mathrm{H}$-band metallicities from low-resolution spectroscopy (Terrien et al. 2012b). We used these stars and performed a detailed analysis between BT-Settl models and the spectra. We compute synthetic spectra over the entire spectral range of interest using a model grid described as follows: $T_{\text {eff }}$ from $2000 \mathrm{~K}$ to $4000 \mathrm{~K}$ with $100 \mathrm{~K}$ steps, $\log g=5.0$ and 5.5 , and $[\mathrm{M} / \mathrm{H}]=-2.0$ dex to +0.5 dex with 0.5 dex steps. We convolve the synthetic spectra with a Gaussian kernel at the APOGEE resolution and then rebin to match the pixel sampling of the observations. As a first step, we calculate a $\chi^{2}$ goodness of fit statistic by comparing the observed spectra with the grids of synthetic spectra. This allows us to estimate effective temperature. Next, assuming this effective temperature we use the most prominent lines in the spectra, the atomic Al lines at $16718 \AA$ and $16750 \AA$, to constrain the other parameters (log $g$ and $[\mathrm{M} / \mathrm{H}])$. The derived parameters are given in Table 3 . The comparison between the observed spectrum (blue) and the best fit model (red) is shown in Figure 10 for 2M19081153+2839105.

We derive stellar parameters using our improved atmosphere models and spectroscopic information covering the given NIR range. Metallicity and gravity are determined from specific spectral features such as $\mathrm{Al}$ I, Fe I, and $\mathrm{CaI}$, whereas effective temperatures are constrained from the overall shape of the spectra via the following steps: (1) a first $\chi^{2}$ minimization is performed on the overall spectra considering effective temperature, metallicity, and gravity as free parameters. It gives a first guess for the parameter space of each component; (2) we look for those specific spectral features that are mainly sensitive to metallicity or gravity to refine these two parameters; and (3) we fixed these parameters to perform another $\chi^{2}$ minimization and derive the effective temperature. At each step we check that the resulting value is not sensitive to changes in the values of the other parameters.

The synthetic spectra reproduce very well most of the spectral features like Fe I, Ca I, and Al I. Some discrepancies remain in the strengths of some atomic lines, which are either too strong or weak in the model, such as Fe I $(15267.02 \AA, 15237.7 \AA$, $15335.00 \AA, 15964.87 \AA$ ) and Ca I (16197.04 $\AA$ ). This is mainly due to the fact that the models have to use some incomplete or approximate input physics, such as broadening damping constants and uncertain oscillator strengths, for some lines and molecular bands. Also, the BT-Settl models use general atomic damping constants according to Unsöld (1968) with a correction 
factor to the van der Waals widths of 2.5 (Valenti \& Piskunov 1996), and van der Waals broadening of molecular lines with generic widths according to Homeier et al. (2003). Furthermore, the BT-Settl models do not have a good handle on modeling the $\mathrm{H}_{2} \mathrm{O}$ bands. This discrepancy is clearly seen in the bottom panel of Figure 10.

Recent progress has been made in the development of empirical calibrations that allow estimation of $[\mathrm{Fe} / \mathrm{H}]$ for M dwarfs to a precision of 0.12-0.15 dex (Rojas-Ayala et al. 2012, 2010; Terrien et al. 2012b). Terrien et al. (2012b) developed such a calibration that uses the equivalent widths of specific regions in $R \sim 2000 \mathrm{H}$-band M-dwarf spectra. This relation is calibrated using a set of $22 \mathrm{M}$-dwarf companions to FGK stars having well-constrained metallicities and by assuming the individual systems are coeval. Column 3 in Table 3 lists the metallicity of three $\mathrm{M}$ dwarfs observed by APOGEE and that have had their metallicity determined using this technique using spectra from the IRTF. The three methods agree with each other within the errors. The more comprehensive comparison study of the metallicity determination through empirical spectroscopic relationships and stellar atmospheric modeling will be explored in a subsequent paper.

The features used for the $H$-band calibration are $\mathrm{K}_{\mathrm{I}}(1.52 \mu \mathrm{m})$ and $\mathrm{Ca} \mathrm{I}(1.62 \mu \mathrm{m})$. These features fall inside the spectral region covered by APOGEE, and so should be amenable to a similar empirical calibration strategy as employed with $R \sim 2000$ spectra used in Terrien et al. (2012b). The results of this empirical metallicity calibration for APOGEE observations of $M$ dwarfs will be presented in a subsequent paper.

\subsection{AO Imaging of $M$ Dwarfs}

We have initiated an extensive campaign to collect AO imaging of a significant sample of $\mathrm{M}$ dwarfs in the APOGEE RV survey in an effort to also detect binaries at wider separations. A number of factors make this attractive and practical.

1. Because they are intrinsically faint, $M$ dwarfs offer less demanding contrast requirements compared to solar-type stars for the direct imaging detection of low-mass companions at small angular separations.

2. M dwarfs in close proximity to the Sun $(d \lesssim 20$ pc) are (nevertheless) sufficiently bright to serve as their own natural guide star.

3. AO instruments offer diffraction-limited performance at NIR wavelengths, facilitating the detection of faint (and red) companions whose blackbody radiation peaks in the $\lambda \approx 1.0-5.0 \mu \mathrm{m}$ range.

4. By concentrating companion light into a compact and locally intense point-spread function, the sensitivity of AO observations benefits from an increased $\mathrm{S} / \mathrm{N}$ compared to seeing-limited or speckle observations.

Large aperture telescopes equipped with AO imagers, such as Palomar (Bouchez et al. 2009), Keck (Wizinowich et al. 2000), and the Large Binocular Telescope (Esposito et al. 2012), provide sensitivity to stellar companions of any mass using only seconds of integration time. High-contrast observations, such as those using AO in combination with a coronagraph and/or pointspread function subtraction (Marois et al. 2006), are sensitive to BDs over essentially all masses and ages with $\approx 1 \mathrm{hr}$ integration times, even at sub-arcsecond separations (Crepp et al. 2012a).

The scientific motivation for combining precision RV measurements with AO observations is equally compelling. By combining two complementary observing techniques, it is possible
Table 4

M-Dwarf Stellar Properties Derived from Model Fits

\begin{tabular}{lcccc}
\hline \hline $\begin{array}{l}\text { Star } \\
(2 \mathrm{MASS})\end{array}$ & $\begin{array}{c}\rho \\
(\mathrm{mas})\end{array}$ & $\begin{array}{c}\text { P.A. } \\
\text { (degrees) }\end{array}$ & $\begin{array}{c}\delta \text { mag } \\
(\mathrm{mag})\end{array}$ & Instrument \\
\hline $03305473+7041145$ & $367.8 \pm 0.2$ & $141.2 \pm 0.2$ & $1.05 \pm 0.05$ & NIRC2 \\
$03425325+2326495$ & $524.7 \pm 0.2$ & $331.9 \pm 0.2$ & $0.24 \pm 0.03$ & NIRC2 \\
$12122940+3940281$ & $470.2 \pm 2.0$ & $340.0 \pm 1.0$ & $0.76 \pm 0.06$ & PHARO \\
\hline
\end{tabular}

to place strong constraints on the presence of both short-period and long-period companions around each star. A joint Doppler and imaging survey will enable detailed studies of stellar multiplicity at the low-mass end of the main sequence. Further, the frequency of BD companions to low-mass stars is still unknown in a large fraction of the parameter space explored by our survey. For instance, Metchev \& Hillenbrand (2009) have quantified the occurrence rate of BDs orbiting FGK stars in the 29-1590 AU range, but similar studies for M-dwarf primaries have only recently commenced (Bowler et al. 2012).

In addition to discovering a plethora of short-period companions, APOGEE's multiplexing capabilities will also reveal systems that exhibit long-term Doppler accelerations indicating the presence of unseen wide-separation companions. RV "trends" act as a signpost to identify promising AO imaging follow-up targets (Crepp et al. 2012b). In the case of a direct detection, multi-epoch AO imaging and continued Doppler measurements can ultimately lead to the construction of three-dimensional orbits and calculation of dynamical masses (Crepp et al. 2012b). Such mass "benchmark" systems may in turn be used to explicitly calibrate theoretical atmospheric models and theoretical evolutionary models of cool dwarfs. Finally, in the case of a non-detection, it is possible to place strong constraints on the mass and period of putative companions (Rodigas et al. 2011; Montet \& Johnson 2013).

Motivated by these factors, we have commenced AO observations of M-dwarf targets in the APOGEE target list starting with the brightest sources. Several candidate companions have been identified using the PALM-3000 AO system at Palomar (Bouchez et al. 2009) and PHARO camera (Hayward et al. 2001) as shown in Figure 11. Given their proximity to the Sun, $\mathrm{M}$ dwarfs in our sample generally have a high proper motion. With 10 mas astrometry precision, consecutive observations separated by only several months may be used to unambiguously determine whether each candidate shares a common proper motion with its host star. First epoch and follow-up AO measurements are on-going. Figure 11 gives examples of some of the M-dwarf binary candidates that have been discovered through AO imaging. Table 4 lists their angular separations, position angles, and delta magnitudes.

\section{DISCUSSION AND FUTURE PROSPECTS}

The APOGEE M-dwarf survey described here will produce a catalog of multi-epoch RV measurements of more than 1400 low-mass stars. These RV measurements are derived from high$\mathrm{S} / \mathrm{N}$, high-resolution $(R \sim 22,500) H$-band spectra gathered as part of an ancillary science program of the main SDSS-III APOGEE survey. These observations will be used to identify individual low-mass spectroscopic binaries, and possibly even short period giant planets. The APOGEE spectra routinely provide velocity precision better than $100 \mathrm{~m} \mathrm{~s}^{-1}$, and we have shown that for bright targets, a detailed analysis of the spectra and the telluric absorption lines they contain can 

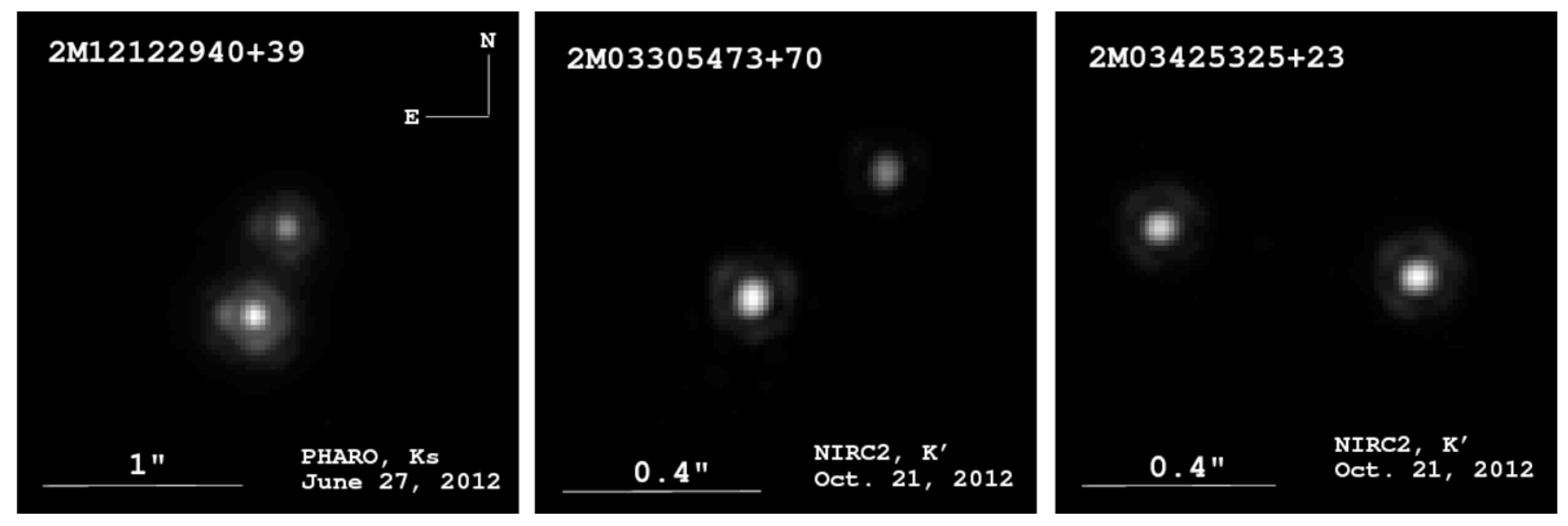

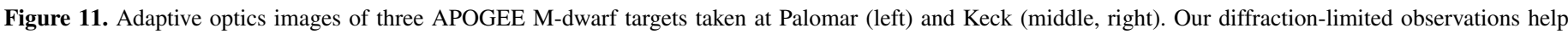
assess the multiplicity of sources by probing separations complementary to RV observations.

result in precision better than $50 \mathrm{~m} \mathrm{~s}^{-1}$. At the same time, these spectra can also be modeled to measure RV, $v \sin i$, and chemical composition. We present $v \sin i$ measurements for over $200 \mathrm{M}$ stars, a significant increase in the total number of available spectroscopic rotation measurements for low-mass stars. Another outcome of this survey is the determination of metallicity through empirical measurements and with the use of stellar atmosphere models such as the BT-Settl models. Combining the results of different analyses of the APOGEE M-dwarf spectra will provide a wealth of information about the structure and evolution of the lowest mass stars.

One of the primary goals of this survey is to quantify the rate of occurrence of companions to $\mathrm{M}$ dwarfs over a wide range of separations and mass ratios. This complete picture of M-dwarf multiplicity will place important observational constraints on theoretical models of the formation of stars at the bottom of the main sequence. We will carry out a joint analysis of wide-separation companions identified through AO imaging and small-separation companions detected as spectroscopic binaries in the APOGEE data. With over 1400 targets in our $\mathrm{RV}$ sample, we expect to detect a large number of binaries, significantly more than any previous single survey. Kinematic measurements derived from these RV data will also be important for placing the local population of $\mathrm{M}$ dwarfs in the context of Galactic stellar populations.

We have carried out a Monte Carlo simulation to estimate our expected sensitivity to short-period companions with a range of masses. We use the actual observational cadence for over $200 \mathrm{M}$ dwarfs observed by APOGEE so far and a simple model for RV precision as a function of magnitude $\left(\sigma_{\mathrm{RV}}=[40,40,66,110,180,300] \mathrm{m} \mathrm{s}^{-1}\right.$ for $H=$ $[7,8,9,10,11,12] \mathrm{mag})$ to simulate $10^{5}$ APOGEE RV surveys. We assume Gaussian noise for stars drawn randomly from the actual $\mathrm{H}$-magnitude distribution (Figure 3 ) and inject RV variations resulting from Keplerian orbits. We assume each star has a mass of $0.4 M_{\odot}$ and consider mass ratios $q=m_{2} / m_{1}$ in the range 0.001 to 1.0 and orbital periods from 1 to 300 days. We select random inclinations, orbital phases, and orbital orientations, and set eccentricity to $e=0$ for periods less than 10 days and $e$ uniformly distributed between 0 and 0.8 for longer periods. For each simulated set of RV measurements of a star we calculate $\chi^{2}$ assuming the null hypothesis of RVs consistent with no variation and count the injected Keplerian orbit as "detected" if the probability of $\chi^{2}$ greater than or equal to the given value is less

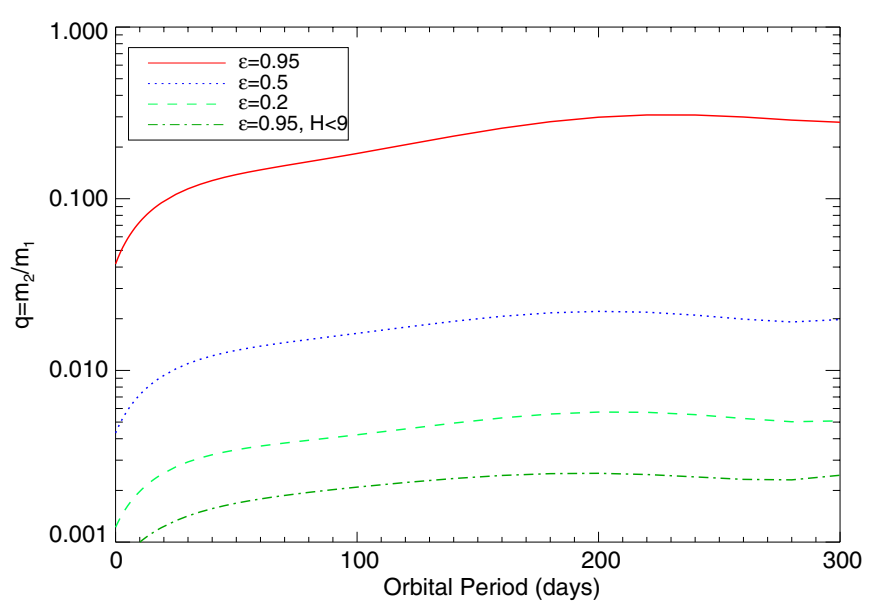

Figure 12. Results of a simulation of detection efficiency. Areas above each line correspond to detection efficiency above a given $\epsilon$. For example, we expect 95\% sensitivity to companions with mass ratios $q>0.25$ and orbital periods less than 300 days (red, solid line).

(A color version of this figure is available in the online journal.)

than $1 \%$. We average the percentage of detections, $\epsilon$, in bins of $q$ and orbital period and show the results of this simulation in Figure 12. Contours corresponding to $95 \%, 50 \%$, and $20 \%$ detection efficiency are shown. We note that the subtle dip in $q$ at long periods is likely an artifact of the time sampling of the APOGEE observations.

Our simulations indicate that the survey will have nearly complete sensitivity to companions with masses greater than $0.08 M_{\odot}$ and orbital periods less than 300 days. At orbital periods less than 10 days, we have excellent sensitivity to companions down to $25 M_{\mathrm{J}}$. For stars brighter than $H=9$ $(N=235)$, we are sensitive to $0.5 M_{\mathrm{J}}$ companions out to orbital periods of 40 days. With this sensitivity and the published occurrence rates for $\mathrm{M}$ dwarf binaries and giant planets orbiting M dwarfs, we expect to detect dozens of new spectroscopic binary systems and place strong limits on the frequency of giant planet companions around $\mathrm{M}$ dwarfs of all spectral types.

Given its large sample size and excellent RV precision, the APOGEE M-Dwarf Radial Velocity Survey will provide a rich data set useful for addressing a number of important outstanding questions about low-mass stars. This survey will generate a large, homogeneous sample of M-dwarf measurements, and 
when completed in 2014, will represent an unprecedented resource useful for studying stellar populations, binary statistics, and measuring physical stellar parameters. At the same time, the physical stellar parameters derived from the APOGEE observations will greatly benefit future planet search surveys such as the Habitable Zone Planet Finder (Mahadevan et al. 2012) and CARMENES (Quirrenbach et al. 2010) by providing detailed information about chemical composition of low-mass stars that are found to host planets. This first paper in a series serves to describe the target selection and present rotational velocities and radial velocities derived from over 1000 individual spectra of more than $200 \mathrm{M}$ dwarfs. The data described in this paper become publicly available as part of the SDSS-III DR10 data release in 2013 July.

This work was partially supported by funding from the Center for Exoplanets and Habitable Worlds. The Center for Exoplanets and Habitable Worlds is supported by the Pennsylvania State University, the Eberly College of Science, and the Pennsylvania Space Grant Consortium. We acknowledge support from NSF grant AST 1006676 and AST 1126413 in our pursuit of precision radial velocities in the NIR. This research has made use of the SIMBAD database, operated at CDS, Strasbourg, France. This publication makes use of data products from the Two Micron All Sky Survey, which is a joint project of the University of Massachusetts and the Infrared Processing and Analysis Center/California Institute of Technology, funded by the National Aeronautics and Space Administration and the National Science Foundation. The authors are visiting astronomers at the Infrared Telescope Facility, which is operated by the University of Hawaii under Cooperative Agreement No. NNX08AE38A with the National Aeronautics and Space Administration, Science Mission Directorate, Planetary Astronomy Program. J.R.C. acknowledges support from NASA Origins grant NNX13AB03G. This research has made use of NASA's Astrophysics Data System.

This work was based on observations with the SDSS $2.5 \mathrm{~m}$ telescope. Funding for SDSS-III has been provided by the Alfred P. Sloan Foundation, the Participating Institutions, the National Science Foundation, and the U.S. Department of Energy Oce of Science. The SDSS-III Web site is http://www.sdss3.org/. SDSS-III is managed by the Astrophysical Research Consortium for the Participating Institutions of the SDSS-III Collaboration including the University of Arizona, the Brazilian Participation Group, Brookhaven National Laboratory, University of Cambridge, Carnegie Mellon University, University of Florida, the French Participation Group, the German Participation Group, Harvard University, the Instituto de Astrosica de Canarias, the Michigan State/Notre Dame/JINA Participation Group, Johns Hopkins University, Lawrence Berkeley National Laboratory, Max Planck Institute for Astrophysics, Max Planck Institute for Extraterrestrial Physics, New Mexico State University, New York University, Ohio State University, Pennsylvania State University, University of Portsmouth, Princeton University, the Spanish Participation Group, University of Tokyo, University of Utah, Vanderbilt University, University of Virginia, University of Washington, and Yale University.

Facilities: Sloan, IRTF.

\section{REFERENCES}

Ahn, C. P., Alexandroff, R., Allende Prieto, C., et al. 2013, arXiv:1307.7735 Allard, F., Hauschildt, P. H., Alexander, D. R., \& Starrfield, S. 1997, ARA\&A, 35,137
Asplund, M., Grevesse, N., Sauval, A. J., \& Scott, P. 2009, ARA\&A, 47, 481 Bailer-Jones, C. A. L. 2004, A\&A, 419, 703

Bean, J. L., Seifahrt, A., Hartman, H., et al. 2010, ApJ, 713, 410

Bender, C. F., \& Simon, M. 2008, ApJ, 689, 416

Blake, C. H., Charbonneau, D., \& White, R. J. 2010, ApJ, 723, 68

Blake, C. H., Charbonneau, D., White, R. J., Marley, M. S., \& Saumon, D. 2007, ApJ, 666, 1198

Blake, C. H., \& Shaw, M. M. 2011, PASP, 123, 1302

Bonfils, X., Delfosse, X., Udry, S., et al. 2013, A\&A, 549, A109

Bouchez, A. H., Dekany, R. G., Angione, J. R., et al. 2009, Proc. SPIE, 7439, 11

Bowler, B. P., Liu, M. C., Shkolnik, E. L., \& Tamura, M. 2012, ApJ, 756, 69

Browning, M. K. 2008, ApJ, 676, 1262

Butler, R. P., \& Marcy, G. W. 1996, PASP, 108, 500

Caffau, E., Ludwig, H.-G., Steffen, M., Freytag, B., \& Bonifacio, P. 2011, SoPh, 268,255

Chabrier, G., \& Baraffe, I. 1997, A\&A, 327, 1039

Chabrier, G., Gallardo, J., \& Baraffe, I. 2007, A\&A, 472, L17

Chubak, C., Marcy, G., Fischer, D. A., et al. 2012, arXiv:1207.6212

Ciardi, D. R., von Braun, K., Bryden, G., et al. 2011, AJ, 141, 108

Claret, A. 2000, A\&A, 363, 1081

Claret, A., Hauschildt, P. H., \& Witte, S. 2012, A\&A, 546, A14

Crepp, J. R., Johnson, J. A., Fischer, D. A., et al. 2012a, ApJ, 751, 97

Crepp, J. R., Johnson, J. A., Howard, A. W., et al. 2012b, ApJ, 761, 39

Delfosse, X., Beuzit, J.-L., Marchal, L., et al. 2004, in ASP Conf. Ser. 318, Spectroscopically and Spatially Resolving the Components of the Close Binary Stars, ed. R. W. Hilditch, H. Hensberge, \& K. Pavlovski (San Francisco, CA: ASP), 166

Deshpande, R., Martín, E. L., Montgomery, M. M., et al. 2012, AJ, 144, 99

Dressing, C. D., \& Charbonneau, D. 2013, ApJ, 767, 95

Duquennoy, A., \& Mayor, M. 1991, A\&A, 248, 485

Eisenstein, D. J., Weinberg, D. H., Agol, E., et al. 2011, AJ, 142, 72

Endl, M., Cochran, W. D., Kürster, M., et al. 2006, ApJ, 649, 436

Endl, M., Cochran, W. D., Tull, R. G., \& MacQueen, P. J. 2003, AJ, 126, 3099 Esposito, S., Pinna, E., Quirós-Pacheco, F., et al. 2012, Proc. SPIE, 8447, 84471L Feiden, G. A., \& Chaboyer, B. 2012, ApJ, 757, 42

Figueira, P., Pepe, F., Melo, C. H. F., et al. 2010, A\&A, 511, A55

Fischer, D. A., \& Marcy, G. W. 1992, ApJ, 396, 178

Freytag, B., Allard, F., Ludwig, H.-G., Homeier, D., \& Steffen, M. 2010, A\&A, 513, A19

Gizis, J. E., Reid, I. N., \& Hawley, S. L. 2002, AJ, 123, 3356

Gomes da Silva, J., Santos, N. C., Bonfils, X., et al. 2012, A\&A, 541, A9

Gray, D. F. 1992, The Observation and Analysis of Stellar Photospheres (2nd ed.; Cambridge: Cambridge Univ. Press)

Grether, D., \& Lineweaver, C. H. 2006, ApJ, 640, 1051

Gunn, J. E., Siegmund, W. A., Mannery, E. J., et al. 2006, AJ, 131, 2332

Hayward, T. L., Brandl, B., Pirger, B., et al. 2001, PASP, 113, 105

Homeier, N. L., Blum, R. D., Pasquali, A., Conti, P. S., \& Damineli, A. 2003, A\&A, 408, 153

Howard, A. W., Johnson, J. A., Marcy, G. W., et al. 2010, ApJ, 721, 1467

Irwin, J., Berta, Z. K., Burke, C. J., et al. 2011, ApJ, 727, 56

Jenkins, J. S., Ramsey, L. W., Jones, H. R. A., et al. 2009, ApJ, 704, 975

Jódar, E., Pérez-Garrido, A., Díaz-Sánchez, A., et al. 2013, MNRAS, 429, 859

Kane, S. R., Henry, G. W., Dragomir, D., et al. 2011, ApJL, 735, L41

Kaüfl, H.-U., Ballester, P., Biereichel, P., et al. 2004, Proc. SPIE, 5492, 1218

Kopparapu, R. K. 2013, ApJL, 767, L8

Lada, C. J. 2006, ApJL, 640, L63

Lafrenière, D., Doyon, R., Marois, C., et al. 2007, ApJ, 670, 1367

Latham, D. W., Stefanik, R. P., Mazeh, T., Mayor, M., \& Burki, G. 1989, Natur, 339,38

Lépine, S., \& Gaidos, E. 2011, AJ, 142, 138

Lépine, S., \& Shara, M. M. 2005, AJ, 129, 1483

Ludwig, H.-G., Allard, F., \& Hauschildt, P. H. 2006, A\&A, 459, 599

Mahadevan, S., Ramsey, L., Bender, C., et al. 2012, Proc. SPIE, 8446, 84461S

Majewski, S. R., Wilson, J. C., Hearty, F., Schiavon, R. R., \& Skrutskie, M. F. 2010, in IAU Symp. 265, Chemical Abundances in the Universe: Connecting First Stars to Planets, ed. K. Cunha, M. Spite, \& B. Barbuy (Cambridge: Cambridge Univ. Press), 480

Marchal, L., Delfosse, X., Forveille, T., et al. 2003, in IAU Symp. 211, Brown Dwarfs, ed. E. Martín (San Francisco, CA: ASP), 311

Marcy, G. W., \& Butler, R. P. 2000, PASP, 112, 137

Marcy, G. W., Cochran, W. D., \& Mayor, M. 2000, in Protostars and Planets IV, ed. V. Mannings, A. P. Boss, \& S. S. Russell (Tucson, AZ: Univ. Arizona Press), 1285

Marois, C., Lafrenière, D., Macintosh, B., \& Doyon, R. 2006, ApJ, 647, 612

Mayor, M., Pepe, F., Queloz, D., et al. 2003, Msngr, 114, 20

McLean, I., Becklin, E. E., Bendiksen, O., et al. 1998, Proc. SPIE, 3354, 566 
McQuillan, A., Aigrain, S., \& Mazeh, T. 2013, MNRAS, 432, 1203

Mermilliod, J.-C., Grenon, M., \& Mayor, M. 2008, A\&A, 491, 951

Metchev, S. A., \& Hillenbrand, L. A. 2009, ApJS, 181, 62

Mohanty, S., \& Basri, G. 2003, ApJ, 583, 451

Montet, B. T., Crepp, J. R., Johnson, J. A., Howard, A. W., \& Marcy, G. W. 2013, arXiv: 1307.5849

Montet, B. T., \& Johnson, J. A. 2013, ApJ, 762, 112

Morin, J., Donati, J.-F., Petit, P., et al. 2008, MNRAS, 390, 567

Nelder, J. A., \& Mead, R. 1965, CompJ, 7, 308

Neves, V., Bonfils, X., Santos, N. C., et al. 2013, A\&A, 551, A36

Noyes, R. W. 1984, AdSpR, 4, 151

Nutzman, P., \& Charbonneau, D. 2008, PASP, 120, 317

Prandtl, L. 1926, Proc. 2nd Int. Congr. Appl. Mech.

Quirrenbach, A., Amado, P. J., Mandel, H., et al. 2010, Proc. SPIE, 7735, 37

Raghavan, D., McAlister, H. A., Henry, T. J., et al. 2010, ApJS, 190, 1

Rajpurohit, A. S., Reylé, C., Schultheis, M., et al. 2012, A\&A, 545, A85

Randich, S., Schmitt, J. H. M. M., \& Prosser, C. 1996, A\&A, 313, 815

Redman, S. L., Lawler, J. E., Nave, G., Ramsey, L. W., \& Mahadevan, S. 2011, ApJS, 195, 24

Redman, S. L., Ycas, G. G., Terrien, R., et al. 2012, ApJS, 199, 2

Reiners, A., \& Basri, G. 2008, ApJ, 684, 1390

Reiners, A., \& Basri, G. 2009, ApJ, 705, 1416

Reiners, A., \& Basri, G. 2010, ApJ, 710, 924

Reiners, A., Joshi, N., \& Goldman, B. 2012, AJ, 143, 93
Rodigas, T. J., Males, J. R., Hinz, P. M., Mamajek, E. E., \& Knox, R. P. 2011, ApJ, 732, 10

Rodler, F., Deshpande, R., Zapatero Osorio, M. R., et al. 2012, A\&A, 538, A141

Rojas-Ayala, B., Covey, K. R., Muirhead, P. S., \& Lloyd, J. P. 2010, ApJL, 720, L113

Rojas-Ayala, B., Covey, K. R., Muirhead, P. S., \& Lloyd, J. P. 2012, ApJ, 748,93

Southworth, J., Pavlovski, K., Tamajo, E., et al. 2011, MNRAS, 414, 3740

Terrien, R. C., Fleming, S. W., Mahadevan, S., et al. 2012a, ApJL, 760, L9

Terrien, R. C., Mahadevan, S., Bender, C. F., et al. 2012b, ApJL, 747, L38

Tonry, J., \& Davis, M. 1979, AJ, 84, 1511

Torres, G. 2013, AN, 334, 4

Torres, G., Clausen, J. V., Bruntt, H., et al. 2012, A\&A, 537, A117

Udry, S., Bonfils, X., Delfosse, X., et al. 2007, A\&A, 469, L43

Unsöld, A. 1968, QJRAS, 9, 294

Valenti, J. A., \& Piskunov, N. 1996, A\&AS, 118, 595

van Eyken, J. C., Ciardi, D. R., von Braun, K., et al. 2012, ApJ, 755, 42

Walkowicz, L. M., Basri, G., Batalha, N., et al. 2011, AJ, 141, 50

Wilson, J. C., Hearty, F., Skrutskie, M. F., et al. 2010, Proc. SPIE, 7735, $77351 \mathrm{C}-1$

Wilson, J. C., Hearty, F., Skrutskie, M. F., et al. 2012, Proc. SPIE, 8446, 84460H

Wizinowich, P., Acton, D. S., Shelton, C., et al. 2000, PASP, 112, 315

Zasowski, G., Johnson, J. A., Frinchaboy, P. M., et al. 2013, AJ, 146, 81 